\title{
INTEGRACIÓN PROPORCIONAL VS MÉTODO DE LA PARTICIPACIÓN: ANÁLISIS DE VALORACIÓN Y CAPACIDAD PREDICTIVA
}

\author{
MÓNICA ESPINOSA (monica.espinosa@ua.es) \\ RAUL IÑIGUEZ (raul.iniguez@ua.es) \\ FRANCISCO POVEDA(fpoveda@ua.es) \\ Departamento de Economía Financiera y Contabilidad \\ Universidad de Alicante \\ Apartado de Correos 99 \\ 03080, Alicante - España \\ Telf. +34965903611 \\ Fax: +34 965903621
}

Revista Española de Financiación y Contabilidad. Forthcoming. 


\title{
RESUMEN
}

La sustitución de la NIC 31 por la NIIF 11, que ha entrado en vigor el 1 de enero de 2013, implica un cambio sustancial en cuanto al método permitido para consolidar las empresas multigrupo. Desaparece la posibilidad de optar por el método de integración proporcional o método de la participación quedando éste último como única alternativa. En este contexto, nos planteamos cómo afecta tal cambio a la calidad de la información contable de las empresas partícipes en empresas multigrupo. A partir del método de elegido por cada empresa española en el periodo 2005-2010, se analiza cuál de los dos procedimientos refleja mejor la imagen fiel de la empresa, conduce a mayor relevancia de la información contable y logra mejores predicciones de beneficios. Los resultados obtenidos sugieren que ambos métodos muestran similar capacidad predictiva y valorativa. Por tanto, no existe suficiente evidencia que apoye una mayor utilidad del método de integración proporcional.

Classification JEL: G14; G32; M41.

Palabras claves: método de consolidación, empresas multigrupo, valoración, predicciones de beneficios

\begin{abstract}
The replacement of NIC 31 by NIIF 11, that becomes effective on a compulsory basis on January $1^{\text {st }}$, 2013 , implies a substantial change in the procedures allowed for the consolidation of the joint ventures. If so far these companies were able to choose voluntarily between proportionate consolidation and the equity method, from now on the first method will be eliminated requiring only the second one. In this context, we bring up if such policy change would lead into a decline in the quality of the accounting information disclosed by Spanish joint ventures in the period 2005-2010, analyzing which of the above mentioned methods better shows the true image of the firm, leads to more relevant accounting information and allows better earnings forecasts. Our results show that there is no evidence in favour of the use of the proportionate consolidation method. Both methods achieve similar forecasting and valuation ability.
\end{abstract}

Classification JEL: G14; G32; M41.

Key words: Consolidation method, joint ventures, valuation, earnings forecasts 


\section{INTEGRACIÓN PROPORCIONAL vs MÉTODO DE LA PARTICIPACIÓN: ANÁLISIS DE VALORACIÓN Y CAPACIDAD PREDICTIVA}

\section{INTRODUCCIÓN}

La consolidación de estados financieros en España se implementa en 1991, con el RD 1915/1991 que introduce las primeras normas de formulación de cuentas anuales consolidadas (en adelante NFCAC). Posteriormente, dichas normas se sustituyen por las vigentes NFCAC que entraron en vigor en 2010, y se desarrollaron en el RD 1159/2010 como continuación a la reforma mercantil que supuso la entrada en vigor del nuevo plan contable en 2008 (en adelante PGC). No obstante, ya desde el 1 de enero de 2005, por imperativo de la Ley $62 / 2003$, se incorpora al ordenamiento jurídico español la obligación de que los grupos de empresas que emitan valores admitidos a cotización en mercados organizados, presenten sus cuentas consolidadas basadas directamente en las NIIF aprobadas en la UE.

En el marco normativo descrito, a la hora de consolidar las empresas multigrupo tanto las NFCAC como las NIIF han permitido la elección voluntaria entre el método de integración proporcional y el procedimiento de puesta en equivalencia (posteriormente referenciado como método de la participación). Esta elección contable ya ha sido suprimida, puesto que la última reforma tramitada por el IASB ha supuesto la desaparición del método de integración proporcional en favor de un único método, el de la participación; aunque con ciertos requerimientos de información adicionales respecto a los activos, pasivos, ingresos y gastos de los negocios conjuntos en los que se participe.

Con relación a la supresión del método de integración proporcional en favor del método de la participación, existen dos matices a tener en cuenta. Por un lado, existe cierta controversia entre la consideración del método de la participación como un método de consolidación propiamente dicho, o un procedimiento de valoración de las participaciones en el negocio conjunto. Hay trabajos como Nobes (2002) que sólo lo consideran un procedimiento de valoración cuando se trata de cuentas individuales, pero que en cuentas consolidadas lo consideran como un método de consolidación. Sin embrago, el debate está presente en la actualidad como puede consultarse en el documento EFRAG (2014) emitido al respecto. Por otro lado, pese a que el método de integración proporcional desaparece como opción, en 
casos muy limitados se aplica una contabilidad de activos y pasivos subyacentes a determinadas empresas multigrupo que no implicaría excesiva variación entre la NIC 31 y la NIIF 11. Concretamente, la nueva clasificación entre actividades conjuntas y negocios conjuntos hace que se extraigan de las entidades controladas de forma conjunta según la NIC 31, aquellos casos en los que, pese a existir un vehículo separado en el diseño formal, prevalece el fondo sobre la forma y se asemejan a los antiguos activos o explotaciones controladas de forma conjunta según la NIC 31. De este modo, lo que antes serían empresas multigrupo por integración proporcional, ahora podrían ser tratadas como actividades conjuntas en la NIIF 11.

La sustitución de la NIC 31 por la NIIF 11, que ha entrado en vigor de forma obligatoria desde el 1 de enero de 2013, implica un cambio de posicionamiento por parte del IASB. Desde el propio organismo se aportan todas las explicaciones pertinentes que tratan de justificar dicho cambio, citando el origen, principalmente en la convergencia internacional. Sin embargo, si nos ceñimos a la última redacción de la NIC 31, la consolidación proporcional se establecía como método preferente, y el IASB expresaba de forma explícita que el método de integración proporcional aportaba más información: "Esta Norma no recomienda el uso del método de la participación, porque considera que la consolidación proporcional refleja mejor el fondo y la realidad económicos de la participación en la entidad bajo control común, es decir, el control sobre su parte de los beneficios económicos futuros. No obstante, esta Norma permite utilizar el método de la participación como un método alternativo al informar sobre las participaciones en entidades controladas conjuntamente."

Desde un punto de vista empírico, según puede consultarse en el apartado segundo del trabajo, no queda claro que el cambio de la NIC 31 a la IFRS 11 vaya a suponer una mejora en la calidad de la información contable, entendiendo por ésta a la comunicación por parte de la empresa de información económico-financiera relevante para una adecuada valoración de la misma en el mercado. A nivel internacional, existe una gran diversidad de estudios centrados en el impacto de la elección del método de consolidación sobre numerosas variables, y los resultados obtenidos son contrapuestos. Además, hasta la fecha no existe ningún trabajo que estudie tales cuestiones para el caso del mercado español. Esta escasa e indeterminada evidencia previa, junto con los últimos cambios normativos acaecidos que hemos argumentado anteriormente, motivan el objeto de nuestra investigación. 
Por ello, el objetivo de nuestro trabajo es analizar si el cambio en la normativa referente a la consolidación de las empresas multigrupo podría traducirse en un cambio en la utilidad de los estados contables de las empresas partícipes para los usuarios de la información financiera. Concretamente, analizamos si la eliminación de la integración proporcional en favor de la puesta en equivalencia tiene algún efecto sobre la relevancia de la información contable, y si lleva a mejores o peores predicciones de beneficios y valoraciones de las acciones de las empresas españolas.

Este análisis se realiza a partir de dos grupos de empresas cuyo factor discriminante es el método de consolidación seleccionado. En otros países, tal y como se comenta en el apartado de revisión de literatura, las empresas partícipes, independientemente del procedimiento de consolidación utilizado, aportan en la memoria información detallada que permite obtener esa misma información contable por el método alternativo. Bajo tales circunstancias, se puede comparar sobre una misma muestra de empresas ambas opciones. Sin embargo, aunque la NIC 31 establece que las empresas revelen los importes de sus activos, pasivos, gastos e ingresos en negocios conjuntos, un análisis preliminar de nuestra muestra indica que este detalle en la información no siempre sucede; de hecho incluso menos del $50 \%$ de las empresas de mayor tamaño realiza dicho desglose. Por ello, en nuestro estudio aplicado a España, las conclusiones acerca de las diferencias entre la integración proporcional y la puesta en equivalencia no proceden de la comparación de las mismas empresas sino de carteras construidas en función del método escogido. La imposibilidad de reconstruir la información comparando empresa a empresa supone una limitación al trabajo que resta potencia a los resultados obtenidos. No obstante, la existencia de limitaciones no resta interés al necesario estudio sobre el posible efecto del cambio normativo sobre la calidad de la información contable en nuestro país.

Para analizar el impacto que ha tenido en el periodo 2005-2010 la utilización del método de integración proporcional y el de la puesta en equivalencia en la valoración de las empresas del mercado español, en primer lugar estimamos el clásico modelo de relevancia valorativa basado en las conclusiones de Ohlson (1995). En segundo lugar, nos basamos en una metodología análoga a la anterior, si bien centrado en la capacidad predictiva de los resultados. La puesta en equivalencia implica una condensación de la información en comparación con la consolidación proporcional, que muestra de manera separada la parte correspondiente de las multigrupo dentro de cada una de las partidas de activos, pasivos, ingresos y gastos. Por tanto, analizamos si dicha desagregación de la información es útil y 
relevante para los usuarios de la información, y por ende, permite realizar mejores predicciones de resultados y valoraciones de empresas.

Nuestros resultados apuntan a que no existen diferencias significativas entre las dos muestras diferenciadas de integración proporcional y método de la participación. Desde el punto de vista de la relevancia valorativa y la capacidad predictiva, ambos métodos parecen mostrarse como equivalentes, por lo que no podemos afirmar que una alternativa sea preferible a la otra. En este sentido, podemos concluir que la supresión del método de integración proporcional como alternativa a elegir por parte de las empresas partícipes en empresas multigrupo no parece suponer una pérdida de utilidad de la información financiera para los usuarios de la misma.

El presente trabajo se estructura como sigue. En el apartado segundo se hace una revisión de los trabajos más relevantes que se han escrito en torno a las causas y consecuencias de la elección contable entre consolidación proporcional y método de la participación. En el apartado tercero se centra el planteamiento empírico a desarrollar así como las hipótesis a contrastar. El apartado cuarto describe el proceso de elaboración de la muestra así como la estadística descriptiva más relevante. En el apartado quinto se presentan los resultados del análisis empírico; y en el sexto apartado se sintetizan las principales conclusiones que pueden extraerse del trabajo.

\section{REVISIÓN DE LITERATURA}

La contabilización de los intereses en negocios conjuntos requiere, según el país y las circunstancias, del empleo del método de la participación o del método de integración proporcional. Numerosos estudios se han centrado en el análisis de los efectos de la adopción de uno de estos dos métodos sobre determinadas variables, tales como la rentabilidad financiera o el riesgo de mercado.

Algunos de los artículos más influyentes se han realizado en el mercado canadiense, ya que este país representa un caso ideal de estudio. Esto se debe a que hasta 1995, the Canadian Institute of Chartered Accountants permitía la elección entre el método de integración proporcional y el de puesta en equivalencia; pero sin embargo a partir de ese año, únicamente la integración proporcional estaba permitida. Graham et al. (2003) encuentran evidencia 
consistente con la visión de que los estados financieros preparados conforme a la integración proporcional de los negocios conjuntos proporcionan mejores predicciones de la futura rentabilidad financiera que los estados financieros elaborados mediante el método de la participación. Kothavala (2003) también obtiene que la integración proporcional proporciona mayor información que el método de la participación a la hora de explicar la volatilidad en los precios de mercado, aunque se obtiene el resultado contrario a la hora de explicar calificaciones crediticias de los bonos emitidos, puesto que en este caso se explican mejor dichas calificaciones con el método de la participación. El autor respalda estos resultados argumentando que la volatilidad en los precios representa un espectro más amplio de los usuarios de la información contable, mientras que la calificación crediticia de los bonos representa un tipo de usuario menos numeroso, pero seguramente más sofisticado. De cualquier manera, nótese que el objetivo de Graham et al. (2003) es investigar la predictibilidad de los resultados, mientras que el de Kothavala (2003) se centra en implicaciones sobre el riesgo percibido.

Richardson et al. (2012) también utiliza una muestra canadiense, pero con el objetivo de investigar las implicaciones de un cambio de normativa en la contabilización de los negocios conjuntos sobre la relevancia valorativa de la información contenida en el balance de situación. Estos autores encuentran evidencia de que las empresas que utilizaban el método de la participación, cuando podía optarse por él, experimentaron una disminución de la relevancia valorativa de los activos y pasivos totales cuando la opción obligatoria pasó a ser la integración proporcional. Sin embargo, las empresas que ya habían optado por la integración proporcional antes de 1995, posteriormente no experimentaron dicha disminución en relevancia valorativa.

Soonawalla (2006) centra su estudio en Canadá y en el Reino Unido, investigando la pérdida potencial en capacidad predictiva y relevancia valorativa al agregar la información sobre empresas multigrupo y asociadas a través del método de la participación. Se utilizan ambos países debido a que, aunque el método utilizado es distinto, en ambos se requiere proporcionar información desagregada que permite llevar a cabo la cuestión de estudio a través del modelo de Ohlson (1999). Sus resultados muestran que la agregación de la información resulta en una pérdida de información que provoca una disminución de la capacidad predictiva y de la relevancia valorativa de la información contable. 
Resulta también interesante referirnos al trabajo de Stoltzfus y Epps (2005), que examina, para una muestra de empresas de Estados Unidos, si la prima de riesgo de los bonos emitidos por las empresas cotizadas están más asociados con la información que proporciona la integración proporcional que la proporcionada por el método de la participación. Estos autores concluyen que los prestamistas no obtienen mejor información con una contabilidad basada en el método de la integración proporcional. No obstante, la integración proporcional sí obtiene una relación más fuerte con la prima de riesgo para aquellas compañías que proporcionan garantías a la deuda. En consonancia con Kothavala (2003), los resultados sugieren que para los negocios conjuntos en los que se garantiza la deuda, la decisión de utilizar el método de la participación en lugar de la integración proporcional, no sería la mejor opción para los intereses de los usuarios de los estados financieros.

O'Hanlon y Taylor (2007) examinan la relevancia valorativa de la información divulgada de acuerdo a lo establecido en la norma contable del Reino Unido FRS 9: Associates and Joint Ventures (ASB, 1997). En el Reino Unido se sigue el método de la participación, pero informando en la memoria de los activos y pasivos de los negocios controlados conjuntamente. Estos autores creen que la divulgación de la información sobre el pasivo de las participaciones en empresas asociadas y negocios conjuntos está negativamente asociada con el valor de mercado de la empresa inversora. Los resultados que obtienen confirman la evidencia previa obtenida por Bauman (2003) en Estados Unidos, de forma que existe un impacto valorativo negativo de la divulgación de los pasivos de las participadas, lo que sugiere que dicha divulgación es percibida como una señal negativa, siendo esta evidencia válida, no sólo para participaciones en negocios conjuntos sino también en empresas asociadas.

Por tanto, del análisis de la literatura previa las conclusiones que se obtienen en cuanto a qué alternativa de consolidación es preferible, son contradictorias. Desde el punto de vista de la relevancia valorativa, estudios como el de Graham et al. (2003) y Soonawalla (2006), consideran preferible el método de integración proporcional para Reino Unido y Canadá. Por el contrario, Bauman (2003) para Estados Unidos, y O'Hanlon y Taylor (2007) para Reino Unido, consideran que la información incremental que aporta la alternativa de integración proporcional es valorada negativamente por parte de los inversores. Así pues, resulta una cuestión abierta e interesante comprobar si en el mercado español la relevancia valorativa y la capacidad predictiva de los resultados futuros puede realizarse de manera significativamente mejor mediante cualquiera de las dos alternativas de consolidación disponibles hasta la entrada en vigor de la NIIF 11. 
Aunque no es nuestro objetivo de investigación, no queremos terminar este apartado sin citar diversos trabajos previos que estudian, no las consecuencias de la elección del método contable de consolidación, sino los determinantes que llevan a las empresas a elegir uno u otro método. Lourenço y Dias (2010) estudian si el tipo de negocio conjunto influye en la elección de los directivos sobre el método de la participación o el de integración proporcional. Concretamente, consideran por un lado, los denominados Scale, en el que las empresas que comparten el negocio conjunto pertenecen a la misma industria (cooperación homogénea). Por otro lado, los denominados Link, donde los partícipes provienen de diferentes industrias y desarrollan un nuevo negocio (cooperación heterogénea). En una muestra de empresas del Reino Unido, los resultados confirman que el tipo de negocio conjunto juega un papel crucial en la decisión del método para reflejar los negocios conjuntos. El método de la participación parece más apropiado para el primer tipo de negocio conjunto, mientras que la integración proporcional parece que se ajusta más al segundo tipo. También se proporciona evidencia de que otros factores resultan relevantes a la hora de decidir la elección del método contable, tales como los costes de acuerdo de financiación, y los costes de control y supervisión.

Más recientemente, y además para el mercado español, Giner y Verón (2012) analizan los factores que han influido en la elección entre el método de la participación y la integración proporcional. En su estudio se encuentra evidencia de que las empresas más grandes, con una concentración no estructurada en torno a accionistas dominantes, menor endeudamiento, con activos más específicos, y con mayor interdependencia entre sus actividades, se inclinan en mayor medida hacia la integración proporcional.

\section{DISEÑO DE LA INVESTIGACIÓN}

\subsection{Hipótesis}

El cambio normativo acaecido tras la sustitución de la NIC 31 por la NIIF 11, conlleva la desaparición de la posibilidad de elección entre el método de integración proporcional y el método de la participación o puesta en equivalencia, para realizar la consolidación de las empresas multigrupo. Este nuevo contexto, en el que el método de la participación es la única alternativa disponible, es el que origina la pregunta de investigación a la que tratamos de dar respuesta en este artículo: cómo afecta tal cambio a la calidad de la información contable.

Para ello, analizamos las consecuencias que para las empresas partícipes tiene la elección contable entre métodos de consolidación para empresas multigrupo, desde la doble 
perspectiva de la relevancia valorativa y de la capacidad predictiva de la información contable, tomando como base una muestra de empresas cotizadas en el mercado de capitales español.

Por un lado, desde el punto de vista de la relevancia valorativa, se analizará la capacidad explicativa de las dos variables contables básicas, patrimonio y resultado, según el clásico modelo de relevancia valorativa basado en Ohlson (1995). De esta forma, se pretende aportar evidencia de si la utilización de uno u otro método de consolidación lleva a diferencias en la capacidad explicativa de los precios observados en el mercado, planteándose la siguiente hipótesis nula:

$H_{1}$ : Los métodos de integración proporcional y puesta en equivalencia proporcionan información con la misma relevancia valorativa.

Por otro lado, se analizará también la capacidad predictiva que la información contable tiene de los resultados con objeto de determinar si el máximo detalle en la información es útil para los usuarios a la hora de realizar predicciones. La hipótesis objeto de contraste es:

$\mathrm{H}_{2}$ : Los métodos de integración proporcional y puesta en equivalencia proporcionan información con la misma capacidad explicativa de los futuros resultados de la empresa.

Una vez analizada la relevancia valorativa y capacidad predictiva con datos históricos reales, proponemos un enfoque alternativo distinto, en el que se realizan predicciones futuras de resultados y valores de la empresa. Dichas predicciones se realizarán con la información disponible que habrá sido elaborada por los partícipes aplicando distintos métodos de consolidación para sus multigrupo. Las predicciones que se realicen de beneficios y las valoraciones intrínsecas de las acciones de la empresa que se estimen se compararán con sus respectivos valores reales observados, obteniéndose unos errores de predicción o valoración. En este sentido las hipótesis nulas a contrastar serían:

$H_{3}$ : Mediante la información proporcionada por las empresas, las predicciones que podemos realizar de los beneficios a 1 año presentan similar precisión, ya hayan optado las empresas partícipes por el método de integración proporcional o por el método de puesta en equivalencia para consolidar sus empresas multigrupo.

$\mathrm{H}_{4}$ : Mediante la información proporcionada por las empresas, las estimaciones que podemos realizar del valor de las acciones de las empresas presentan similar precisión, ya hayan optado las empresas partícipes por el método de integración proporcional o por el método de puesta en equivalencia para consolidar sus empresas multigrupo. 


\subsection{Planteamiento y metodología}

Para analizar el impacto de la utilización de un método u otro sobre la valoración de las empresas cotizadas en el mercado español, en primer lugar estimamos el clásico modelo de relevancia valorativa basado en las conclusiones de Ohlson (1995), en el que las dos variables contables básicas, patrimonio neto y resultado, son los determinantes principales del valor de mercado de las acciones de la empresa:

$P_{i t}=\alpha_{1}+\alpha_{2} b v_{i t}+\alpha_{3} x_{i t}+\varepsilon_{i t}$

donde:

$P_{i t}$ : Precio de mercado total de las acciones de la empresa i en el momento $t$;

$b v_{i t}$ : Valor en libros o patrimonio neto de la empresa i en el momento $t$;

$\mathrm{x}_{\mathrm{it}}$ : resultado contable de la empresa i en el ejercicio t;

El modelo anterior lo estimamos para toda la muestra disponible y también de forma separada en cada una de las dos submuestras que vienen dadas por el método de consolidación escogido en cada grupo. Se trata de comparar los coeficientes del modelo de valoración en sección cruzada entre las empresas que consolidan por integración proporcional y las que lo hacen siguiendo el método de la participación.

Para poder contrastar la significatividad de la diferencia entre ambos coeficientes estimados, introducimos variables binarias en la parte de las variables explicativas estableciendo como categoría base el método de integración proporcional (valor binario igual a cero). De este modo, los coeficientes que se obtienen en las variables binarias son precisamente la diferencia entre los coeficientes de la categoría base (integración proporcional) y los de la categoría diferenciada (puesta en equivalencia):

$P_{i t}=\alpha_{1}+\alpha_{11} P P E_{i t}+\alpha_{2} b v_{i t}+\alpha_{21} b v_{i t} P P E_{i t}+\alpha_{3} x_{i t}+\alpha_{31} x_{i t} P P E_{i t}+\varepsilon_{i t}$ donde:

$\mathrm{PPE}_{\mathrm{it}}$ : variable binaria que toma el valor 0 si la empresa $\mathrm{i}$ en el ejercicio $\mathrm{t}$ optó por la integración proporcional para consolidar las multigrupo; y el valor 1 si optó por el procedimiento de puesta en equivalencia;

$\alpha_{2}$ y $\alpha_{3}$ : coeficientes de valoración de las empresas que optan por la integración proporcional; $\left(\alpha_{2}+\alpha_{21}\right)$ y $\left(\alpha_{3}+\alpha_{31}\right)$ : coeficientes de valoración de las empresas que optan por la puesta en equivalencia;

$\alpha_{21}$ y $\alpha_{31}$ : diferencial entre los coeficientes de valoración de ambas submuestras. 
La hipótesis nula a contrastar, $\mathrm{H}_{1}$, es la referente a la igualdad de coeficientes de valoración entre las submuestras de integración proporcional y puesta en equivalencia, ya que sería indicativo de que ambos métodos presentan la misma relevancia valorativa. En caso de que la integración proporcional diera lugar a información más relevante, cabe esperar un coeficiente de determinación $R^{2}$ superior en la submuestra de integración proporcional (ecuación [1]). Además, calculamos el Criterio de la Información de Akaike (AIC, Akaike Information Criterion, véase Akaike 1974). AIC es una medida de la bondad de ajuste de un modelo estadístico que se utiliza como herramienta para la selección de modelos, ofreciendo una medida relativa de la pérdida de información de un modelo, por lo que se considera como mejor modelo aquel que obtiene el menor valor AIC. No obstante, debemos advertir que AIC no proporciona un contraste en términos de hipótesis nula, ni cuánto de bueno o de malo es un modelo, sino simplemente cuál es mejor.

En segundo lugar, analizamos la capacidad predictiva de los resultados. Debemos tener en cuenta que la puesta en equivalencia implica una condensación de la información en una única valoración, mientras que en la consolidación proporcional aparece la parte correspondiente dentro de cada una de las partidas de activos, pasivos, gastos e ingresos. Si dicha desagregación es relevante y útil para los usuarios de la información, o dicho de otro modo, permite conocer mejor la situación financiera actual y futura de la empresa así como de sus resultados, entonces deberíamos observar un mejor ajuste en una regresión en la que se predice el futuro resultado a partir de los datos actuales. Por ello, tratamos de analizar la capacidad predictiva de la información contable a través de los siguientes modelos:

$x_{i t+1}=\alpha_{1}+\alpha_{2} b v_{i t}+\alpha_{3} x_{i t}+\varepsilon_{i t}$

$x_{i t+1}=\alpha_{1}+\alpha_{11} P P E_{i t}+\alpha_{2} b v_{i t}+\alpha_{21} b v_{i t} P P E_{i t}+\alpha_{3} x_{i t}+\alpha_{31} x_{i t} P P E_{i t}+\varepsilon_{i t}$

donde además de las variables antes definidas, $x_{i t+1}$ : resultado contable de la empresa $i$ en el ejercicio $t+1$.

Debemos decir que la expresión [3] se utiliza, entre otros, en Soonawalla (2006). A la hora de anticipar el resultado del ejercicio siguiente, cabe esperar que un mayor detalle de los gastos e ingresos que componen la cifra de resultados, frente a una cifra única, aporte mayor capacidad predictiva. Por ello, la hipótesis nula a contrastar, $\mathrm{H}_{2}$, es la referente a la igualdad de coeficientes de predicción entre las submuestras de integración proporcional y puesta en equivalencia, ya que sería indicativo de que ambos métodos tienen la misma capacidad predictiva. En caso de que la integración proporcional diera lugar a información más relevante, 
cabe esperar un coeficiente de determinación $\mathrm{R}^{2}$ superior en la submuestra de integración proporcional, así como un menor valor del AIC.

Con relación al método de estimación de todos los modelos anteriores, ante los problemas de escala que suelen atribuirse a este tipo de modelos (véase, por ejemplo Easton y Sommers (2003), y para asegurar la robustez de los resultados, tenemos en cuenta tres procedimientos ${ }^{1}$ :

- Deflactando todas las variables en niveles por la capitalización bursátil. Este procedimiento es equivalente a la propuesta de Easton y Sommers (2003), que recomiendan la estimación por Mínimos Cuadrados Ponderados donde se utiliza para ponderar el valor de mercado de la compañía en t-1.

- La metodología utilizada en Hand (2005), que consiste en someter a las variables en niveles a una transformación logarítmica que suavice la incidencia de los valores elevados y mitigue sensiblemente las interferencias del efecto escala en los resultados.

- Técnicas de datos de panel sin deflactar los datos, concretamente a través de un modelo de efectos fijos. El modelo de efectos fijos supone tener en cuenta la heterogeneidad de las distintas empresas de la muestra, y equivale a estimar un modelo con tantos interceptos como unidades empresariales existen en la muestra.

Los dos primeros métodos de estimación empleados permiten sortear las críticas expuestas por Holthausen y Watts (2001) en torno a la utilización de los niveles de las variables, puesto que estamos utilizando un deflactor en el primer caso y una transformación directa en el segundo. En el tercer caso, utilizamos las técnicas de datos de panel sin deflactar las variables, lo que permite no cambiar el sentido económico de los modelos y su interpretación, que es la principal crítica que se le hacen a las soluciones que popularmente se han utilizado para solventar el problema del efecto escala.

Por otro lado, dado que los coeficientes son estimados en un pool de datos, los estadísticos de contraste pueden estar sobredimensionados debido a la ausencia de independencia entre las observaciones (una misma empresa aparece en varios años). Por ello, los errores estándar de las regresiones los hemos calculado haciendo clusters por empresa y año de acuerdo con la propuesta de Petersen (2009).

\footnotetext{
${ }^{1}$ Desgraciadamente, la literatura no ha encontrado una solución óptima al efecto escala, existiendo diversas propuestas, siendo las más utilizadas la deflactación por una variable que aproxime el tamaño de la empresa (capitalización bursátil, total activo, etc.), o el uso de datos por acción. Dado que no existe una solución universalmente aceptada, para dar mayor robustez a nuestro estudio hemos optado por utilizar tres soluciones muy distintas entre sí.
} 
Si bien el análisis de regresión descrito arriba ha sido la metodología usualmente utilizada en los estudios previos, en nuestro estudio queremos dar un paso más. Por ello, vamos a incorporar el cómputo de predicciones de resultados y su posterior utilización para la valoración de las acciones de las empresas, mediante el uso de modelos comúnmente utilizados en la literatura contable y financiera. Una vez estimados los futuros resultados y valores, comparamos dichas predicciones con los valores que realmente se observan, obteniéndose los errores de predicción o valoración cometidos mediante la información disponible, que habrá sido elaborada, en lo que respecta a las empresas multigrupo, aplicando distintos métodos de consolidación.

\section{A) Predicciones de resultados futuros}

Utilizamos varias especificaciones para predecir futuros resultados. Concretamente:

Modelo 1. Modelo del recorrido aleatorio. El resultado esperado de una empresa para el próximo ejercicio es justo el resultado del periodo actual.

$$
E_{t}\left[x_{i t+1}\right]=x_{i t}
$$

Modelo 2. Modelo con tendencia lineal temporal. Los resultados de una empresa siguen una evolución lineal creciente o decreciente conforme va pasando el tiempo. En primer lugar, se regresa el resultado sobre la variable tiempo:

$$
x_{i t}=\propto+\beta \cdot t+e_{t}
$$

Realizándose posteriormente la siguiente predicción sobre el resultado de la empresa:

$$
E_{t}\left[x_{i t+1}\right]=\widehat{\alpha}+\hat{\beta} \cdot(t+1)
$$

Modelo 3. Modelo autorregresivo de orden 1. Los modelos $A R(1)$ son muy conocidos y utilizados en cualquier contexto de series temporales. Se basa en el hecho de que parece sensato pensar que el beneficio de un año dependa del obtenido en el año anterior, pudiéndose obtener una pauta de evolución en la observación de su evolución en el pasado. Se estima en primer lugar el modelo autorregresivo:

$$
x_{i t}=\alpha+\beta \cdot x_{i t-1}+e_{t}
$$

Que da lugar a la siguiente predicción:

$$
E_{t}\left[x_{i t+1}\right]=\widehat{\alpha}+\hat{\beta} \cdot x_{i t}
$$


Modelo 4. Modelo autorregresivo de orden 2. Si añadimos un retardo adicional al modelo anterior, nos encontramos con el también conocido modelo $A R(2)$, en el que el beneficio actual es función de los beneficios de los dos últimos ejercicios. Se trata de la siguiente regresión:

$$
x_{i t}=\propto+\beta \cdot x_{i t-1}+\gamma \cdot x_{i t-2}+e_{t}
$$

Que da lugar a la siguiente predicción del resultado futuro:

$$
E_{t}\left[x_{i t+1}\right]=\widehat{\alpha}+\hat{\beta} \cdot x_{i t}+\hat{\gamma} \cdot x_{i t-1}
$$

Modelo 5. Modelo autorregresivo del resultado con tendencia temporal. En este caso, proponemos combinar un modelo autorregresivo $\mathrm{AR}(1)$ con un modelo con tendencia temporal:

$$
x_{i t}=\propto+\beta \cdot t+\gamma \cdot x_{i t-1}+e_{t}
$$

Realizándose la predicción del resultado como sigue:

$$
E_{t}\left[x_{i t+1}\right]=\widehat{\alpha}+\hat{\beta} \cdot(t+1)+\hat{\gamma} \cdot x_{i t}
$$

Modelo 6. Modelo autorregresivo del resultado que incluye el patrimonio neto como variable relevante. Por último, tenemos en cuenta una predicción basada en $\operatorname{AR}(1)$, incluyendo el patrimonio neto como variable relevante para la estimación del beneficio a un año, que es el equivalente a la ecuación [3] del análisis de relevancia del subapartado anterior:

$$
x_{i t}=\propto+\beta \cdot x_{i t-1}+\gamma \cdot b v_{i t-1}+e_{t}
$$

Siendo en este caso la predicción del resultado del próximo ejercicio:

$$
E_{t}\left[x_{i t+1}\right]=\widehat{\alpha}+\hat{\beta} \cdot x_{i t}+\hat{\gamma} \cdot b v_{i t}
$$

La estimación de los modelos anteriores puede hacerse bien como una serie temporal de secciones cruzadas (todas las empresas intervienen en la regresión), bien en serie temporal (una única regresión por empresa). Utilizar una sección cruzada tiene la ventaja de poder utilizar el máximo tamaño muestral, al no requerirse un mínimo de datos en serie temporal para realizar las estimaciones. A cambio, se pierde información específica de cada empresa que podría ser útil para realizar las predicciones. Por el contrario, estimar en serie temporal explota al máximo la información específica de cada empresa en concreto, pero al necesitarse un mínimo de extensión de la serie temporal, se pierden las observaciones de los primeros 
ejercicios. En nuestro estudio aplicaremos ambos métodos de estimación, serie temporal y sección cruzada.

En el caso de sección cruzada, las estimaciones se realizan tanto para toda la muestra (mismos coeficientes para todas las empresas de la muestra) como separadamente para cada submuestra; es decir, calculando distintos coeficientes para el subgrupo de empresas que han optado por la integración proporcional y el subgrupo de empresas que ha optado por la puesta en equivalencia. En este sentido cabe mencionar las ventajas e inconvenientes de ambos procedimientos. En el caso de estimaciones utilizando la muestra completa, puede que exista cierto sesgo en el estudio, ya que si hay más empresas que utilizan un método de consolidación frente al otro, es posible que los coeficientes estimados se ajusten mejor para el grupo de empresas más numeroso. Esto puede provocar que realmente no se aprecie correctamente si la información obtenida de las empresas que utilizan el método menos utilizado tiene más relevancia valorativa que la emanada del método de consolidación elegido por la mayoría de empresas. Si, por el contrario, se estima el valor de los parámetros en sección cruzada para cada submuestra, el proceso parecería más lógico. Sin embargo, si hay un subgrupo mucho menos numeroso que otro, puede que las estimaciones sean menos fiables, por lo que los errores de predicción pueden depender crucialmente del grado de bondad de las estimaciones de los parámetros. Por ello, decidimos utilizar ambos procedimientos con objeto de proporcionar al estudio de mayor robustez y así poder valorar en mayor medida el grado de bondad de las estimaciones y conclusiones obtenidas.

En cuanto a series temporales, para solventar el inconveniente de pérdida de observaciones, descargamos de la base de datos Compustat Global Vantage todos los beneficios de las empresas de la muestra desde el año 1991 al 2004. Esta serie temporal completa nuestro periodo muestral 2005-2010 a efectos de estas regresiones individuales por empresa, evitando la pérdida de numerosas observaciones. No obstante, y para asegurar cierta consistencia en las estimaciones, una empresa sólo pasará a formar parte de esta parte del estudio en serie temporal si como mínimo existen 5 años consecutivos de beneficios.

Con cualquiera de los métodos, una vez estimadas las regresiones y calculadas las predicciones para cada empresa y año, calculamos el error absoluto de las predicciones a 1 año de cada una de las observaciones. Esto es, para corroborar lo precisa o imprecisa que es una estimación debemos analizar la diferencia entre el resultado realmente conseguido por la empresa en el periodo de predicción $(t+1)$, y la predicción que hemos realizado con los modelos P1 a P6 para 
dicho periodo, predicción que ha sido realizada utilizando la información disponible hasta el periodo t. Además, para que el distinto tamaño de cada sociedad no influya en los resultados, este error debe ser deflactado. Por ello, calculamos el error absoluto de predicción de cada observación, empresa i en periodo t, como:

Error Absoluto Predicción $n_{i t}=\left|\frac{x_{i t+1}-E_{t}\left[x_{i t+1}\right]}{\text { Deflactor }}\right|$

Donde $x_{i t+1}$ es el resultado realmente obtenido por la empresa en el periodo $t+1 ; y E_{t}\left[x_{i t+1}\right]$ es el resultado que ha sido estimado a partir de los modelos [P1] a [P6], ya sea con estimaciones en corte transversal o series temporales. Nótese que se toman valores absolutos para evitar obtener un Error de Predicción muy pequeño debido a cuantiosos errores positivos y negativos, que en promedio se compensan entre ellos.

Una vez tenemos calculados los errores absolutos de predicción para cada empresa y periodo conforme a la expresión [5], es usual calcular la media o la mediana de dichos errores para obtener el error medio o mediano absoluto de predicción. Para evitar la influencia de valores extremos o atípicos en los errores calculados nos decantamos por presentar nuestros resultados en términos medianos ${ }^{2}$.

En cuanto al deflactor, Leung (2011) apunta varias alternativas: una variable representativa del tamaño de la empresa (por ejemplo, la capitalización bursátil); la propia variable que se predice (el resultado, ya sea la predicción o el dato real); o seguir la propuesta de Makridakis (1993), esto es, tomar la media del dato real y la predicción del resultado.

En nuestro estudio, puesto que un beneficio o predicción cercana a cero implica un valor muy pequeño en el denominador que puede afectar a las conclusiones, creemos conveniente utilizar la primera opción. Por ello, utilizar como deflactor una variable identificativa del tamaño de la empresa, concretamente la capitalización bursátil ${ }^{3}$. La hipótesis nula a contrastar, la $\mathrm{H}_{3}$, se refiere a la equivalencia en la precisión de las predicciones de beneficios que se realicen mediante el uso de la información proporcionada por las empresas, que habrá

\footnotetext{
${ }^{2}$ Las conclusiones que sacaremos de este apartado no cambian si observamos los valores medios en lugar de los medianos.

${ }^{3}$ Igualmente, como análisis de sensibilidad hemos probado las otras dos opciones, y las conclusiones de nuestro estudio se mantienen.
} 
sido elaborada siguiendo diferente método de contabilización de las participaciones en empresas multigrupo.

\section{B) Valoración intrínseca de las acciones de una empresa}

En este subapartado contrastamos la capacidad valorativa en ambos subgrupos de empresas, las que han optado por la integración proporcional y las que han optado por la puesta en equivalencia. En este caso, los errores de valoración se calculan como sigue:

Error Absoluto Valoración $=\left|\frac{P_{i t}-V_{i t}}{\text { Deflactor }}\right|$

Donde $\mathrm{P}_{\mathrm{it}}$ es el precio de mercado real de la empresa $\mathrm{i}$ en el periodo $\mathrm{t}+1$; $\mathrm{y} V_{\mathrm{it}}$ es el valor intrínseco estimado en el momento $t$ a partir de los modelos [V1] a [V5] que vemos a continuación.

El deflactor que utilizamos es, de nuevo, el de la opción i, esto es, la capitalización bursátil ${ }^{4}$. Y la hipótesis nula a contrastar en este caso sería la $\mathrm{H}_{4}$, que se refiere a la equivalencia en las estimaciones del valor de las acciones realizadas mediante el uso de la información contenida en los estados contables, se hayan realizado aplicando uno u otro método para la consolidación por parte del partícipe de sus empresas multigrupo.

Los modelos concretos que utilizaremos para realizar la valoración son diferentes versiones del modelo de Ohlson (1995) tal y como se justifica a nivel teórico en numerosos trabajos como el de Larrán y Piñeiro (2005).

Modelo 1. Valoración en base al balance o activos netos. Este modelo determina el valor de una empresa como el valor de sus activos netos, ya sea por su valor contable, su valor de mercado, su valor de reposición, su valor sustancial o su valor de liquidación (véase, entre otros, Fernández 2004, y Pricewaterhouse Coopers 2003). En nuestro caso lo aproximamos por su valor contable, esto es, el patrimonio neto $\left(b_{\mathrm{it}}\right)$ dividido por el número de acciones:

$$
V_{i t}=\frac{b v_{i t}}{N^{\mathrm{o}} \text { acciones }_{i t}}
$$

Modelo 2. Valoración en base a la cuenta de resultados o beneficios. En este caso el valor viene dado por el valor actual de los beneficios proyectados futuros. En caso de suponer una duración infinita de la empresa, el valor es (Pricewaterhouse Coopers 2003, p. 114):

\footnotetext{
${ }^{4} \mathrm{Al}$ igual que antes, otros deflactores no llevan a conclusiones diferentes en nuestro estudio.
} 


$$
V_{i t}=\frac{x_{i t}}{r}
$$

Donde $r$ es el coste de capital o tasa de descuento.

Modelo 3. Modelo mixto basado en el patrimonio neto y el resultado residual. Uno de los modelos que mejor resultado está dando en las investigaciones realizadas en los últimos años, es el modelo del resultado residual, popularizado a raíz del modelo de Ohlson (1995). El resultado residual obtenido por una empresa se define como (véase Ohlson 1995):

$x_{i t}^{a}=x_{i t}-r \cdot b v_{i t-1}$

Donde $x_{i t}^{a}$ es el resultado anormal o residual de la empresa i en el periodo $t, x_{i t}$ es el resultado del ejercicio $t$, $r$ el coste de capital y bvit-1 el patrimonio neto a inicios del periodo.

Diversos estudios han comparado su precisión con otros modelos comúnmente utilizados en la práctica, obteniéndose su superioridad frente a modelos como el de flujos de caja o el de descuento de dividendos (véase, por ejemplo, Penman y Sougiannis 1998, Francis et al. 2000). Para su aplicación a efectos valorativos, una vez obtenido el resultado anormal puede demostrarse que el valor de la empresa sería su patrimonio neto más el valor actualizado de todos los resultados residuales esperados futuros. Simplificando, suponiendo que el resultado residual actual se va a mantener a perpetuidad:

$$
V_{i t}=b v_{i t}+\frac{x_{i t}^{a}}{r}
$$

Hay que tener presente que los modelos, [V2] y [V3] requieren la utilización de una tasa de descuento o coste de capital. Utilizamos un tipo de interés variable en el tiempo pero constante entre empresas, concretamente el tipo de interés a 1 año de las letras del tesoro ${ }^{5}$ más una prima por riesgo del $6 \%$.

Modelo 4. Modelo mixto basado en el patrimonio neto y el resultado contable. Este modelo establece que el valor de la empresa depende de las dos variables contables básicas: el patrimonio neto contable y el resultado, que tienen una importancia diferente según el tipo de empresa de que se trate. La justificación teórica a esta especificación econométrica se puede encontrar en Rees (1997):

\footnotetext{
${ }^{5}$ El tipo de interés de las letras del tesoro a 12 meses se ha tomado de la Sección Estadísticas de la web del Banco de España (www.bde.es). Concretamente la serie BE 2116.13, tipo medio ponderado de las letras del tesoro a 1 año. ${ }^{6}$ Dechow et al. (1999) también optan por una tasa constante, en su caso, del $12 \%$. En cuanto a la prima de riesgo, su elección la justificamos en Marín y Rubio (2001, p. 300), que encuentran una prima por riesgo del 6,77\% entre 1963 y 1997, tomando como cartera de mercado el Índice General de la Bolsa de Madrid. Por otro lado, el Documento no 7 de Valoración de Empresas de AECA (2005, Anexo 3) hace mención a un 5,24\% para el periodo 1980-2004. Un 6\% es un valor intermedio entre ambas estimaciones.
} 
$V_{i t}=\alpha_{1}+\alpha_{2} b v_{i t}+\alpha_{3} x_{i t}+\varepsilon_{i t}$

Se considera que si la empresa tiene beneficios y espera seguir teniéndolos, el peso de la valoración recae sobre el resultado contable; pero si la empresa tiene pérdidas, el resultado se considera transitorio (García Ayuso et al. 1999). Para aplicar este modelo necesitamos los coeficientes de valoración $\alpha_{1}, \alpha_{2}, y \alpha_{3}$. Una vez estimados el valor de la empresa es:

$V_{i t}=\hat{\alpha}_{1}+\hat{\alpha}_{2} b v_{i t}+\hat{\alpha}_{3} x_{i t}$

Modelo 5. Modelo mixto basado en OhIson (2001). Ya que utilizamos modelos de valoración derivados del modelo del resultado residual, resulta especialmente interesante incorporar una valoración basada en la especificación econométrica que propone el propio Ohlson (2001):

$V_{i t}=\alpha_{1}+\alpha_{2} b v_{i t}+\alpha_{3} x_{i t}+\alpha_{4} d_{i t}+\alpha_{5} x_{i t+1} \varepsilon_{i t}$

Donde todas las variables han sido definidas anteriormente, salvo $d_{i t}$, que representa los dividendos del periodo $t$.

Para aplicar el modelo y obtener una valoración de cada empresa en cada periodo, estimamos todos los coeficientes $\alpha_{i}$. Tras dicha estimación, obtenemos el valor de cada empresa en cada periodo a través de la siguiente expresión $n^{7}$ :

$V_{i t}=\hat{\alpha}_{1}+\hat{\alpha}_{2} b v_{i t}+\hat{\alpha}_{3} x_{i t}+\hat{\alpha}_{4} d_{i t}+\hat{\alpha}_{5} x_{i t+1}$

De manera análoga a lo comentado en los modelos de predicción de beneficios, la estimación del valor según los modelos [V4] y [V5] requiere la estimación de los coeficientes $\alpha_{i}$. Dicha estimación la realizamos individualmente para cada empresa a través de un modelo de serie temporal; y en sección cruzada. En este último caso, consideramos los dos casos posibles: estimaciones para toda la muestra (mismos coeficientes para todas las empresas de la muestra), y estimaciones para cada submuestra (distintos coeficientes para el subgrupo de empresas que han optado por la integración proporcional y el subgrupo de empresas que ha optado por la puesta en equivalencia).

Nótese que nuestro objetivo a lo largo de todo este apartado no es encontrar el mejor y más preciso modelo de valoración, por ello, hemos optado por modelos sencillos y populares, lejos

\footnotetext{
${ }^{7}$ Una limitación en la aplicación práctica de este modelo de Ohlson (2001) es que es necesario disponer del resultado del periodo $t+1$. Hemos optado en nuestra aplicación empírica por utilizar el dato ex-post, es decir, utilizar el verdadero beneficio obtenido en el siguiente ejercicio. Hand y Landsman (2005), Penman y Sougiannis (1998) y García-Ayuso y Monterrey (1998) también utilizan datos ex-post en sus aplicaciones del modelo de Ohlson o del modelo del resultado residual.
} 
de las complejidades derivadas de estimaciones, de betas, de horizontes finitos de predicción, de valores terminales, etc., que nos llevaría a complejas valoraciones que dependen de la elección de otros variables que nada tienen que ver con la elección contable del método de consolidación de las participaciones en empresas multigrupo. Tampoco utilizamos modelos de cash-flows o dividendos, puesto que estos modelos se basan en flujos que apenas dependen del método contable elegido para contabilizar las participaciones en multigrupo.

Tampoco queremos finalizar este apartado de metodología sin dejar de resaltar una limitación impuesta por la disponibilidad de datos en nuestro estudio: las empresas partícipes en empresas multigrupo proporcionan la información de sus cuentas anuales en base al método de consolidación que hayan elegido, esto es, integración proporcional o puesta en equivalencia; no siendo posible siempre obtener la información financiera de una misma empresa en base a los dos procedimientos alternativos, ya que en numerosos casos las empresas españolas no proporcionan en la memoria la información sobre los activos, pasivos, gastos e ingresos de sus participaciones. De esta forma, a la hora de realizar las regresiones anteriores, y de comparar la capacidad predictiva de cada modelo, hay que tener en cuenta que las submuestras de integración proporcional y de puesta en equivalencia están formadas por empresas diferentes. Por este motivo, dentro del análisis empírico se hacen subcarteras por tamaño y nivel de endeudamiento que permiten homogeneizar los rasgos básicos de las empresas comparadas maximizando así la probabilidad de que las posibles diferencias se deban al método de consolidación aplicado.

\section{MUESTRA Y DATOS: ANÁLISIS DESCRIPTIVO}

A la hora de implementar desde un punto de vista empírico nuestro estudio, nos hemos centrado en el periodo 2005-2010, abarcando desde el primer ejercicio en el que, de acuerdo con la Ley 62/2003, se aplicaron las NIIF en las cuentas consolidadas de las cotizadas españolas. Para este periodo se ha descargado la información contable necesaria para implementar nuestro estudio de todas las empresas cotizadas en España que está disponible en la base de datos Compustat Global Vantage. Seguidamente, en base a las cuentas anuales registradas en la CNMV y disponibles a través de su página web, se han descargado y revisado todas las memorias de cada ejercicio para comprobar el método de consolidación escogido en presencia de empresas multigrupo. La muestra inicial bruta es de 147 empresas, pero dado que sólo son de utilidad en el presente estudio aquellas empresas que presenten cuentas consolidadas en las que existan empresas multigrupo, esta limitación nos deja con 100 
empresas. Dado que nuestro estudio abarca un periodo de 6 años (2005 a 2010), el número máximo de observaciones asciende a 600.

Ahora bien, no todas las empresas están presentes en cada uno de los seis años que abarca el periodo muestral, por varios motivos: bien porque han empezado a cotizar más tarde del año 2005; bien porque dejaron de hacerlo antes de finalizar el ejercicio 2010; bien porque no en todos los años existiera alguna multigrupo en sus cuentas. Concretamente, de los 600 casos teóricamente posibles, nos encontramos 88 faltas por los motivos, por lo que el número de observaciones se reduce a 512. Por último, para formar parte del estudio exigimos como mínimo la disponibilidad, simultáneamente, de las siguientes variables: precio, patrimonio neto y resultado. Tenemos 24 faltas de al menos una de estas tres variables, por lo que el número de observaciones (empresa/año) final asciende a 488. Por ello, el máximo de observaciones que aparece en las tablas 1 y 2 asciende precisamente a dicho número, que es el correspondiente a las variables básicas imprescindibles para llevar a cabo el estudio ${ }^{8}$.

A continuación realizamos un mero análisis descriptivo de las tablas, en las que además hacemos una serie de comparaciones entre las dos submuestras de empresas que han optado por uno u otro método de consolidación. No debe entenderse este análisis meramente descriptivo en ningún caso como una búsqueda de diferencias o determinantes de la elección contable, puesto que nuestro objetivo se centra en las consecuencias de la elección.

La tabla 1 muestra el número de observaciones y el valor medio de una serie de variables tanto para la muestra total, como para cada una de las submuestras que vienen dadas por el método de consolidación aplicado a las empresas multigrupo. En la última columna de la tabla se muestra el p-valor de un contraste de diferencia de medias. La tabla 2 responde al mismo patrón pero utilizando medianas como medida de posición central robusta, y contraste no paramétrico de la diferencia entre las medianas de las submuestras en la última columna.

\section{[INSERTAR TABLA 1 POR AQUÍ]}

\section{[INSERTAR TABLA 2 POR AQUÍ]}

\footnotetext{
${ }^{8}$ No obstante, como puede observarse en las tablas 1 y 2, algunas variables concretas de interés secundario no estaban disponibles en su totalidad, concretamente hay 1 empresa en la que no está disponible el total activo, los dividendos distribuidos y los ingresos por lo que las variables correspondientes disponen de sólo 487 observaciones. En 10 casos no se dispone del número de acciones, reduciéndose pues a 478 las observaciones del beneficio por acción. Y en 24 casos no se dispone de una serie temporal suficiente como para calcular la beta, por lo que está variable está disponible en sólo 464 casos.
} 
Dada la sistemática aplicada en cada método de consolidación, en la integración proporcional el activo y el endeudamiento del balance consolidado incorporan la parte de dichas partidas correspondiente a las empresas multigrupo participadas. Sin embargo, en la puesta en equivalencia sólo aparecen valoradas las participaciones por su valor neto. En cuanto al patrimonio neto, al restar ambas magnitudes, el importe final entre ambos métodos es equivalente. En principio, ceteris paribus, cabe esperar que la rentabilidad económica (ROA) fuera superior en las empresas con puesta en equivalencia pues a igualdad de resultado, la ausencia de los activos de la multigrupo en el denominador hacer subir la ratio.

Si comparamos entre submuestras en sección cruzada las primeras ratios de la tabla 1 , observamos que, efectivamente, la ROA media en puesta en equivalencia es superior a la de integración proporcional. No obstante, la diferencia sólo es significativa al 7\%; y además en términos de medianas (tabla 2) dicha diferencia desaparece.

En cuanto a la rentabilidad financiera (ROE), como decíamos, debería mantenerse constante entre los distintos métodos. Esto no ocurre en media, aunque sí en mediana por lo que parece ser que la existencia de valores atípicos está afectando a los datos de la tabla 1, no así a los de la tabla 2, al ser únicamente en términos de posición central (mediana) ${ }^{9}$. En mediana, no existen diferencias significativas en el valor en libros, ni en el resultado antes de impuestos y, por tanto, no queda soportada la existencia de diferencias en la ROE.

Con relación a la ratio entre deuda y activo, no existe una predicción clara sobre su dirección en la comparativa entre submuestras pues la integración proporcional sesga al alza tanto numerador como denominador. Dependiendo de donde impacte más en términos relativos pueden hacer subir o bajar la ratio. Se observa un mayor endeudamiento de las empresas que aplican integración proporcional.

Nos centramos ahora en el cociente entre la deuda y el valor en libros. Dado que esta última variable no se ve afectada por el método de consolidación, cabe esperar que, en igualdad de condiciones, el mayor endeudamiento generado por el método de consolidación proporcional provoque mayores ratios. En media y media se observa que la ratio de endeudamiento es significativamente superior en la submuestra de integración proporcional.

\footnotetext{
${ }^{9}$ Debido a la presencia de observaciones extremas el estudio se realizará windsorizando las variables que intervienen en las regresiones. Además, como análisis de sensibilidad de nuestro estudio, se ha truncando las variables a sus percentiles 1-99 sin que tampoco varíen los resultados en ningún caso.
} 
Más allá de las ratios, las medidas en niveles permiten contrastar los resultados esperados dado el funcionamiento de ambos métodos. Centrándonos directamente en la tabla 2 para obviar el impacto de observaciones atípicas, hemos advertido ya que la submuestra de empresas que utiliza la integración proporcional tiene mayores niveles de activo. Esto no debe interpretarse necesariamente como empresas de mayor tamaño, ya que puede tratarse del propio efecto provocado por la propia elección contable (la integración proporcional implica un aumento del activo en comparación con la puesta en equivalencia). Efectivamente, si medimos el tamaño en base a su capitalización bursátil o su valor en libros (no se ve afectado por la aplicación de uno u otro método), no existen diferencias significativas de medianas.

Tal vez una de las variables que pueda aportar información interesante sea la de la rentabilidad por dividendos, calculada como el cociente entre los dividendos distribuidos durante el ejercicio y el precio de la acción al final del periodo. Esta variable permite relacionar los trabajos que tratan de asociar la elección contable del método de consolidación multigrupo con el objetivo que la empresa inversora persigue en dichas participadas (véase Hennart 1988, y Lourenço y Dias 2010). La hipótesis es que en aquellos casos en los que la intención de la empresa inversora sea la de involucrarse en el desarrollo de las actividades de la multigrupo, existe respaldo económico para defender que se incorpore la proporción de activos y de deuda que se asume y, por tanto, justifica la aplicación del método de integración proporcional. Sin embargo, en aquellos casos en los que la intención de la empresa inversora sea la obtención de dividendos sin involucrarse activamente en la gestión, tal vez tiene más sentido económico su tratamiento por puesta en equivalencia. Con esta perspectiva, puede comprobarse que la rentabilidad por dividendos en la submuestra de empresas en las que se aplica la puesta en equivalencia es significativamente superior tanto en media como en mediana.

En cuanto al resto de variables que aparecen en las tablas 1 y 2 (ingresos, beneficio por acción, precio, ratio market-to-book y beta) los resultados conjuntos no muestran evidencia robusta de diferencias entre la submuestra de empresas que ha optado por integración proporcional y la submuestra de empresas que ha optado por la puesta en equivalencia.

Para finalizar este análisis descriptivo, y puesto que en el apartado de Diseño de la Investigación hemos optado por utilizar distintos modelos de predicción basados en series temporales y tendencias temporales, hemos realizado un análisis preliminar de las series temporales de beneficios de las empresas de la muestra, así como su tendencia en el tiempo. 


\section{[INSERTAR TABLA 3 POR AQUÍ]}

Concretamente, realizamos regresiones en serie temporal para cada empresa individual por separado a través de un modelo $A R(1)$, un modelo $A R(2)$, y un modelo de tendencia temporal. Puesto que hemos optado por utilizar distintas soluciones al efecto escala que supone utilizar las variables tanto en valores absolutos como deflactados, mostramos el análisis temporal tanto del resultado en términos absolutos (sin deflactar), como del resultado deflactado por la capitalización bursátil.

En el panel A se muestran los coeficientes medios y medianos para la muestra en su totalidad, que son resultado de las estimaciones con cada modelo para cada empresa individual por separado. Se observa como los coeficientes $\alpha_{1}$ estimados son significativos para más de la mitad de la muestra, y que la persistencia del resultado es alta (aproximadamente 0,77 para el resultado sin deflactar y 0,57 para el resultado deflactado). Los $R^{2}$ también resultan elevados, si bien superiores en los modelos autorregresivos que en el modelo de tendencia temporal. Añadir un segundo retardo aumenta ligeramente el $R^{2}$ de la regresión, aunque los coeficientes $\alpha_{2}$ estimados sólo son significativos para algo menos de un $25 \%$ de la muestra. Por lo que parece que los resultados tienen una "memoria" más bien corta. En cualquier caso, parece que los modelos considerados son capaces de detectar en una gran parte de las empresas la tendencia de la serie.

Los paneles B y C muestran los resultados para cada submuestra, en la que se observan resultados muy similares, si bien en la muestra de puesta en equivalencia el porcentaje de coeficientes significativos y el $\mathrm{R}^{2}$ son ligeramente superiores para los modelos autorregresivos, pero ligeramente inferiores para el modelo de tendencia temporal.

\section{ANÁLISIS EMPÍRICO Y RESULTADOS}

A continuación detallamos los resultados de nuestro estudio. En la tabla 4 se muestran los resultados de la estimación de los modelos [1] y [2], correspondientes al análisis de relevancia valorativa. En el panel A la regresión se realiza por Mínimo Cuadrados Ordinarios deflactando por la capitalización bursátil, en el panel B mediante transformación logarítmica de las variables, y en el panel $C$ con datos de panel, método de efectos fijos. La primera fila de cada 
panel hace referencia a la estimación para todas las observaciones, mientras que las dos siguientes son la estimación para la submuestra de integración proporcional y puesta en equivalencia, respectivamente. La última fila muestra la estimación para un modelo con dummies (siendo la categoría base, dummy igual a cero, la integración proporcional) que mide la variación del coeficiente de puesta en equivalencia respecto del coeficiente base de integración proporcional. Dado que el método de integración proporcional, en principio, revela mayor información, si dicha información es relevante y útil para que los usuarios de la información puedan conocer y predecir mejor la situación financiera de la empresa y de sus resultados, en principio cabe esperar que la diferenciación entre ambos subgrupos de empresas lleve a un mayor $\mathrm{R}^{2}$ o un menor valor del Criterio de la Información de Akaike (AIC).

\section{[INSERTAR TABLA 4 POR AQUÍ]}

En la tabla 4 se puede observar como la elección de un método de consolidación u otro apenas tiene impacto en los coeficientes de valoración. De hecho, la segregación de coeficientes a través de variables binarias aporta un coeficiente de determinación muy similar al del resto de estimaciones. Centrándonos en el resultado contable, el coeficiente estimado para la submuestra de puesta en equivalencia es superior al de integración proporcional, aunque dicha diferencia, medida por el coeficiente $\alpha_{31}$ no es estadísticamente significativa (paneles B y C), o lo es sólo al 10\% (panel A). Este resultado sugiere una igualdad entre ambos métodos contables, integración proporcional y puesta en equivalencia.

En cuanto al coeficiente del valor en libros, sí parece que es inferior para la submuestra de empresas con puesta en equivalencia, lo que iría en la línea del resultado obtenido por Graham et al. (2003) para el mercado canadiense. No obstante, la diferencia medida por el coeficiente $\alpha_{21}$ sólo es significativa estadísticamente al 5\% en los paneles B y C. Los resultados por tanto, a nivel de significatividad de los coeficientes, muestran que prácticamente no existen diferencias entre la utilización del método de integración proporcional o de puesta en equivalencia a la hora de analizar la relevancia valorativa.

Por otro lado, los $\mathrm{R}^{2}$ ajustados no muestran diferencias significativas; es más, en el panel $\mathrm{A}$ numéricamente la muestra de puesta en equivalencia consigue ligeramente un mayor $R^{2}$, mientras que en los paneles $\mathrm{B}$ y $\mathrm{C}$ es la muestra de integración proporcional la que mejora por un escaso margen su $\mathrm{R}^{2}$. En cuanto a la bondad de ajuste medida por el Criterio de la Información de Akaike, en los tres paneles aparece como mejor modelo (menor AIC) el de la 
submuestra de puesta en equivalencia. Es más, la comparación entre la regresión con toda la muestra sin diferenciar subgrupos de empresas (primera línea de cada estimación) y la regresión con dummies (última línea) que sí diferencia ambas submuestra es clara: el $\mathrm{R}^{2}$ apenas mejora (se mantiene en paneles A y B, y aumenta de 0,33 a 0,34 con datos de panel), y el AIC apenas varía (1,54 a 1,55 en el primer caso, 2,73 a 2,72 en el segundo, y 1,27 a 1,25 en el tercero).

En definitiva, a efectos de comparar la relevancia valorativa de los datos contables, y en base a la información disponible en nuestro país, no podemos más que concluir que no existe evidencia suficiente como para poder afirmar que un procedimiento de consolidación de las multigrupo supere al otro, aceptándose la hipótesis nula de igualdad de relevancia entre los métodos de integración proporcional y puesta en equivalencia.

A continuación, en la tabla 5 se muestran los resultados de las regresiones de las expresiones [3] y [4], en las que evaluamos la capacidad predictiva del beneficio a 1 año mediante el uso de las dos variables fundamentales contables: patrimonio neto y resultado.

\section{[INSERTAR TABLA 5 POR AQUÍ]}

En los tres paneles y con distintas soluciones al efecto escala, se obtienen resultados parecidos $^{10}$. Como cabe esperar, el resultado actual es útil para predecir el resultado del próximo año, ya que el coeficiente $\alpha_{3}$ es estadísticamente significativo al $1 \%$ en todos los casos y submuestras, salvo uno. El coeficiente asociado al patrimonio neto $\left(\alpha_{2}\right)$ también resulta de utilidad, aunque en el panel A sólo al 10\% para la muestra IP, no siendo significativa para la muestra de puesta en equivalencia (PPE). No obstante, si nos centramos directamente en la estimación del modelo de predicción con variables dummy por método de consolidación (última línea de cada panel de la tabla 5) se puede comprobar que, de nuevo, los coeficientes asociados al resultado y al patrimonio neto de las empresas que optan por uno u otro método son iguales. Esto se comprueba en los coeficientes $\alpha_{21} y \alpha_{31}$, que no son significativamente diferentes de cero a niveles convencionales, salvo en el panel $C$, que el del resultado es significativo aunque sólo al $10 \%$.

\footnotetext{
${ }^{10}$ La transformación logarítimica implica la generación de variables con diferentes varianzas y covarianzas respecto de las variables deflactadas por lo que se desaconseja la comparación directa entre los coeficientes obtenidos en los diferentes paneles. Ocurre exactamente lo mismo con los coeficientes de determinación tal y como se explica en Gu (2007).
} 
Por tanto, de los resultados se desprende que la significatividad de las variables resultado actual y patrimonio neto es similar de cara a la predicción del beneficio a 1 año para las empresas partícipes en empresas multigrupo, independientemente del método de consolidación que hayan seleccionado.

En cuanto al $R^{2}$, se observa un mayor coeficiente en la submuestra de integración proporcional con respecto a la de puesta en equivalencia en los paneles $A$ y $B$, pero la diferenciación de coeficientes no conlleva absolutamente a ninguna mejora en el $\mathrm{R}^{2}$. Así la primera línea de ambos paneles muestra que el $R^{2}$ de toda la muestra es de 0,39 (panel A) y 0,76 (panel B). Si diferenciamos ambas submuestras, los resultados son idénticos en términos de $R^{2}$ (última línea de cada panel). En términos de AIC, se observan similares conclusiones, observándose valores inferiores de AIC para la muestra de integración proporcional, si bien las diferencias son inapreciables en transformación logarítmica. La comparación entre el modelo sin diferenciar coeficientes y diferenciando coeficientes son inexistentes y no en la misma dirección (pequeña disminución y mejora en panel A, 1,58 a 1,57; pequeño aumento y empeoramiento en panel $B$, $2,92$ a 2,93$)$.

En el panel $C$ se mantienen similares conclusiones, con un $\mathrm{R}^{2}$ ligeramente superior y un AIC inferior en la muestra de puesta en equivalencia, pero diferencias escasísimas entre el modelo [3] y [4] tanto en términos de $R^{2}$ como de AIC. Por tanto, las conclusiones se mantienen conforme a lo visto en el análisis de relevancia valorativa, en el sentido de que en base a la información disponible en nuestro país no existe suficiente evidencia que apoye una diferenciación entre las dos submuestras. Los resultados sugieren que se acepta la hipótesis nula de igualdad de relevancia predictiva, ya que no se observa ninguna ventaja explicativa en los modelos al considerar el método de consolidación elegido para las empresas multigrupo.

La tabla 6 muestra los resultados sobre los errores absolutos de predicción de las estimaciones de beneficios a 1 año, realizadas utilizando los modelos [P1] a [P6] que pueden consultarse en el apartado 3. En el panel A aparece el modelo [P1], que es un simple modelo de recorrido aleatorio que no necesita ninguna estimación de coeficientes. En el panel B los modelos [P2] a [P6], que requieren la estimación previa de los coeficientes $\alpha$ señalados en dicho apartado 3. Una vez obtenidos estos coeficientes estimados, se estiman los beneficios a un año y se calculan los errores de predicción en valor absoluto. 
El análisis realizado diferencia entre las empresas según hayan optado por la integración proporcional o la puesta en equivalencia. Además, también diferencia según las predicciones se hayan realizado con estimaciones en sección cruzada o serie temporal ${ }^{11}$. Concretamente, la primera columna del panel B recoge las predicciones realizadas con estimaciones de los coeficientes en sección cruzada (mismos coeficientes para todas las empresas de la muestra); la segunda columna también en sección cruzada, pero diferentes coeficientes para cada submuestra de elección del método de consolidación; y la tercera columna con estimaciones por empresa en serie temporal. El número de errores calculados (no tabulado) varía entre los modelos, oscilando entre un mínimo de 355 del modelo [P4], que requiere dos retardos, a un máximo de 453 del [P1], que es el modelo que menos información requiere. La muestra de integración proporcional ronda las 300 observaciones, por las aproximadamente 100 de la muestra de puesta en equivalencia.

\section{[Insertar Tabla 6 por aquí]}

Si nos fijamos en la magnitud del error mediano absoluto cometido por ambos subgrupos, sólo en el modelo [P1], panel A, el error es menor para la integración proporcional, si bien la diferencia no es significativa. Para el resto de modelos del panel B, se comete el menor error en las empresas que han optado por la puesta en equivalencia, salvo alguna excepción. Ahora bien, en sección cruzada las diferencias sólo son significativas al $5 \%$ en dos de los modelos, el [P2] de tendencia temporal, y el [P3] de tipo $A R(1)$, y este último únicamente diferenciando coeficientes por submuestras. En los restantes se acepta la hipótesis nula de igualdad de errores.

Si nos fijamos en las estimaciones en serie temporal, los errores cometidos son inferiores a los de sección cruzada, como cabe esperar al estimar coeficientes individualizados por empresa. En este caso no existen diferencias entre ambas submuestras en 5 de los modelos, siendo numéricamente inferiores los errores en algunos casos para la puesta en equivalencia y en algunos casos para la integración proporcional. La única diferencia relevante al 5\% se produce de nuevo en el modelo [P2], siendo favorable a las empresas que han elegido la puesta en equivalencia.

\footnotetext{
${ }^{11}$ Tal y como ya comentamos en el apartado de Planteamiento e Hipótesis, aunque nuestro estudio se centra en el periodo 2005-2010, que es el periodo en el que hemos analizado las cuentas anuales para comprobar el método de consolidación empleado por cada empresa de la muestra, hemos obtenido de Compustat Global Vantage los datos contables necesarios desde el año 1991 para poder realizar estimaciones de series temporales más largas.
} 
En definitiva y teniendo en cuenta los datos de los que disponemos en nuestro país, los resultados no muestran diferencias significativas en los errores cometidos al predecir el beneficio a 1 año de las sociedades con empresas multigrupo a través de distintos modelos y procedimientos de estimación. Esto implica aceptar la hipótesis nula de similar precisión en las estimaciones de los beneficios a un año. Todo lo contrario, de encontrarse diferencias, sería ligeramente favorable a la puesta en equivalencia, no pudiendo concluir que la integración proporcional sea mejor alternativa capaz de proporcionar información de mayor utilidad para sus usuarios.

En cuanto a la capacidad valorativa de la información proporcionada con cada uno de los métodos, la tabla 7 muestra los resultados de los errores absolutos de valoración cometidos con cada uno de los modelos. La estructura de la tabla es análoga a la de la tabla 6 anterior. En esta ocasión, los tres primeros modelos no requieren estimaciones de coeficientes y aparecen en el panel A. Sin embargo, los modelos [V4] y [V5] sí necesitan dichas estimaciones, por lo que aparecen en el panel B, según la forma de estimarse los coeficientes: en sección cruzada con toda la muestra, sección cruzada de manera separada por submuestra de método elegido de consolidación, y estimaciones individuales en serie temporal.

Los resultados encontrados son muy similares a los de las predicciones de resultados. En todos los modelos y casi todos los casos, la evidencia es favorable a la puesta en equivalencia, aunque por muy estrecho margen, lo que hace que las diferencias no sean significativas. Tan sólo en el modelo [V2], en el que el valor depende del beneficio actual capitalizado a perpetuidad se encuentra una diferencia significativa al $5 \%$, siendo favorable a la puesta en equivalencia. Por tanto, se acepta la hipótesis nula de igualdad de precisión en las valoraciones, independientemente del método elegido para consolidar las multigrupo.

En definitiva, y teniendo en cuenta que en España no es posible disponer de los datos financieros de las empresas utilizando las dos alternativas de consolidación, hemos obtenido unos resultados que parecen mostrar que ambos métodos de consolidación presentan similar capacidad valorativa, por lo que la decisión de aplicar en todos los casos la puesta en equivalencia a partir de 2013 no parece que redunde en una pérdida de la capacidad predictiva y valorativa de los estados financieros. Estos resultados contrastan con los obtenidos por Graham et al. (2003) y Soonawalla (2006), para Reino Unido y Canadá, respectivamente, donde la integración proporcional se muestra como la alternativa preferible. 


\section{[Insertar Tabla 7 por aquí]}

Una cuestión importante que podría argumentarse es la necesidad de controlar por problemas de endogeneidad. Tal y como demuestran, Giner y Verón (2012), la elección del método contable viene influida por una serie de determinantes. Estos determinantes podrían afectar a los resultados obtenidos. Para comprobar este aspecto, consideramos dos de las variables de Giner y Verón, aquellas que además habitualmente se toman como medida de control en la literatura contable: el tamaño y el endeudamiento.

A continuación replicamos las tablas 6 y 7 desagregando los resultados en dos carteras de tamaño y dos carteras de endeudamiento ${ }^{12}$. En cada submuestra la división en dos carteras se realiza asignando idéntico número de observaciones a cada cartera, de modo que, por ejemplo, en la cartera de IP + deuda, estarán las empresas que han optado por integración proporcional y tienen un endeudamiento superior a la mediana de empresas que han optado por dicho método de consolidación. En la cartera IP - Deuda estarán las restantes empresas, que lógicamente tendrán un endeudamiento inferior a la mediana.

Al igual que Giner y Verón (2012), como tamaño optamos por el total activo, mientras que como endeudamiento tomamos el cociente entre Deuda y Activo. En la tabla 8 se presentan los resultados en las predicciones por método elegido para las multigrupo, según si la empresa está dentro del grupo de empresas grandes o pequeñas, o dentro del grupo de empresas más o menos endeudadas. La tabla 9 muestra los resultados análogos pero para los errores de valoración.

Por cuestiones de espacio, el detalle de la información proporcionada en las tablas no es tan pormenorizado como las tablas 6 y 7 . En lugar de mostrar los errores de cada submuestra y modelo, mostramos directamente la diferencia entre los errores de la muestra de integración proporcional y los errores de la muestra de puesta en equivalencia, de forma que si la diferencia entre ambas es positiva, se cometen más errores en las empresas que han optado por la integración proporcional. Si la diferencia es negativa, se cometen más errores en las empresas que han utilizado la puesta en equivalencia. Para establecer si dicha diferencia es

\footnotetext{
${ }^{12}$ El motivo de hacer sólo dos carteras se debe a que la muestra de puesta en equivalencia no es muy numerosa.
} 
significativa hacemos uso de los símbolos estándar $(* * *, * *, *)$ que identifican diferencias significativas al $1 \%, 5 \%$ y $10 \%$ respectivamente.

\section{[Insertar Tabla 8 por aquí]}

Si observamos los resultados de la tabla 8, no se observan diferencias en las submuestras de elección contable por tamaño. Así, para el modelo 1 (panel A), las diferencias en los errores absolutos de predicción de las empresas más grandes es prácticamente cero (-0,058 favorable para la integración proporcional), lo mismo que en las más pequeñas $(0,0052$ favorable para la puesta en equivalencia). Diferencias no significativas a niveles convencionales. Si observamos el panel B los resultados son muy similares. Si nos fijamos en los números, casi siempre las diferencias son positivas, lo que implica menores errores absolutos en la muestra de puesta en equivalencia. Sólo en 7 casos las diferencias son significativas, en 4 de ellos sólo al 10\%, y en los restantes 3 al 5\%. En dichos casos la evidencia es favorable también a la puesta en equivalencia, y en 5 de ellos ocurren en la submuestra de empresas más grandes. Nótese que casi todos los casos de diferencias significativas ocurren en las estimaciones en corte transversal, mientras que en serie temporal la evidencia es mucho más fuerte a favor de la igualdad entre ambos métodos de consolidación.

Así pues, la evidencia sugiere que no existen diferencias significativas por tamaño, pero de haberlas, de nuevo la evidencia es favorable ligeramente al procedimiento de puesta en equivalencia, reafirmando la conclusión de que la adopción de este método no aparenta una pérdida de la capacidad predictiva de la información financiera ${ }^{13}$.

En cuanto al endeudamiento, los resultados son similares a los del tamaño. Así, en corte transversal la evidencia es favorable a la puesta en equivalencia para las empresas más endeudadas (menos errores en las empresas que han optado por la puesta en equivalencia y tienen un endeudamiento superior a la mediana), pero en serie temporal o en el modelo [P1] que no requiere estimaciones, las diferencias prácticamente desaparecen, sólo manteniéndose al 10\% para el modelo [P2]; y al 5\% para el [P5]. Así pues aunque no existe suficiente evidencia

\footnotetext{
${ }^{13}$ Se ha repetido el mismo análisis tomando la capitalización bursátil como proxy del tamaño. Las conclusiones básicamente se mantienen: evidencia algo más favorable a la puesta en equivalencia en estimaciones en corte transversal (si bien es más acusada en las empresas grandes), sin diferencias en las estimaciones en serie temporal.
} 
robusta, de nuevo parece ser que los resultados, de ser favorables, lo son para la puesta en equivalencia ${ }^{14}$.

\section{[Insertar Tabla 9 por aquí]}

En la tabla 9 observamos el análisis análogo, pero para los errores absolutos de valoración, desglosando por método contable de consolidación y endeudamiento y tamaño. Por tamaño, la evidencia es dispar. En los modelos [V4] y [V5] no se observan diferencias entre los subgrupos, y numéricamente hay signos positivos y negativos. En los modelos [V1] a [V3] sí existe evidencia al $5 \%$ de diferencias para la muestra de empresas de menor tamaño, pero en un caso es favorable a la integración proporcional y en dos a la puesta en equivalencia ${ }^{15}$. Por endeudamiento, las diferencias entre las submuestra de método de consolidación son prácticamente inexistentes, sólo de manera aislada se observa alguna diferencia significativa al $10 \%$, favorable a la puesta en equivalencia en el modelo [V2] y [V4], y una favorable a la integración proporcional en el modelo [V5] para las empresas menos endeudas.

En definitiva, los resultados no siguen una pauta clara y sistemática que permitan sacar conclusiones sobre la superioridad de un método sobre otro, ni sobre el hecho de que el endeudamiento y el tamaño estén determinado la evidencia encontrada en las tablas 4 a 7. A partir de los datos disponibles en España, los resultados indican que parece existir una igualdad entre ambos métodos de consolidación, y de haber alguna diferencia, sería favorable a la puesta en equivalencia, por lo que la utilidad de la información contable en lo referente a capacidad predictiva y valorativa no parece que se vaya a ver alterada por la supresión del método de integración proporcional.

\section{CONCLUSIONES}

El objetivo fundamental de nuestro trabajo es analizar si la elección entre el método de integración proporcional o el de puesta en equivalencia a la hora de consolidar las cuentas de

\footnotetext{
${ }^{14}$ Se ha repetido el mismo análisis tomando el cociente entre Deudas y Patrimonio Neto como proxy del endeudamiento. Las conclusiones se mantienen, si bien la evidencia no es tan favorable a la puesta en equivalencia como la aquí presentada, ya que en serie temporal no se observa absolutamente ninguna ventaja hacia este método.

${ }^{15}$ Tomando la capitalización bursátil como medida del tamaño los resultados son análogos, si bien se observan incluso menos diferencias entre los subgrupos.
} 
las empresas multigrupo, provoca diferencias en la calidad de la información proporcionada, que se traduzca a su vez en diferencias en cuanto a la capacidad de predicción y valoración futura.

La sustitución de la NIC 31 por la NIIF 11, cuya entrada en vigor se ha producido el 1 de enero de 2013 elimina la posibilidad de elección voluntaria entre el procedimiento de la puesta en equivalencia o método de la participación y la integración proporcional para las empresas multigrupo. A partir de ahora, el IASB establece como único método para la consolidación de estas empresas el método de la participación.

Ante la cambiante situación en la que nos encontramos actualmente, y debido a la incertidumbre que se plantea en cuanto a si tal decisión supone o no una pérdida en la calidad de la información contable, nos planteamos el presente trabajo, en el que tratamos de aportar evidencia actual en el mercado español acerca de si el cambio de normativa va a suponer un empeoramiento en la imagen fiel de la empresa, va a conducir a una menor o mayor relevancia de la información contable y va a lograr mejores o peores predicciones de beneficios y valoraciones de acciones.

Como ya se ha señalado, nuestro trabajo cuenta con una importante limitación: las empresas partícipes en empresas multigrupo proporcionan la información de sus cuentas anuales en base al método de consolidación que hayan elegido, esto es, integración proporcional o puesta en equivalencia; pero en un gran porcentaje de los casos no desglosan de manera separada los activos, pasivos, ingresos y gastos en negocios conjuntos. Por ello, no es posible obtener la información financiera en base a los dos procedimientos alternativos, por lo que únicamente dispondremos de esos datos por un método u otro, no siendo directamente comparables las submuestras de integración proporcional y de puesta en equivalencia. Esta situación no se da en otros países donde independientemente del método seleccionado las empresas partícipes muestran información en la memoria que permite obtener la versión de las cuentas anuales por el método de consolidación alternativo. Tal escenario conlleva que el presente trabajo no gire en torno a una única muestra de empresas cuyos datos financieros se han obtenido por partida doble, sino que disponemos de dos grupos de empresas que se diferencian precisamente por la alternativa de consolidación escogida.

Nuestro trabajo es novedoso en dos aspectos. En primer lugar, se trata del primer estudio centrado en las consecuencias de la elección en el mercado español. Y en segundo lugar, en 
cuanto al alcance de tales consecuencias; nuestro estudio es pionero al analizar si la elección del método de la participación o el de integración proporcional conlleva una diferencia en la calidad de la información proporcionada que provoque una mejor o peor predicción y valoración futura.

Por un lado se analiza el impacto de la utilización de un método u otro sobre la valoración de las empresas cotizadas a partir del modelo de relevancia valorativa basado en las conclusiones de Ohlson (1995). El análisis se plantea tanto para la muestra en su conjunto como para las dos submuestras confeccionadas a partir del método de consolidación escogido, y considerando distintas soluciones metodológicas a la hora de estimar los distintos modelos. Así se utilizan tres soluciones distintas al efecto escala, y se realizan las estimaciones tanto en sección cruzada como en serie temporal. La hipótesis nula objeto del contraste es la igualdad de coeficientes de valoración entre las submuestras de integración proporcional y puesta en equivalencia. Los resultados parecen indicar que la elección entre un método de consolidación u otro apenas tiene impacto en los coeficientes de valoración. Por tanto, no existe evidencia suficiente que permita afirmar que un método de consolidación sea preferible al otro, aceptándose la hipótesis nula de igualdad de relevancia entre ambos métodos.

Por otro lado analizamos la capacidad predictiva de los resultados. La puesta en equivalencia implica una condensación de los datos en el balance y en la cuenta de resultados; frente a la consolidación proporcional, que aporta una mayor desagregación. Si tal desagregación es relevante y útil para los usuarios de la información permitiendo un mejor conocimiento de la situación financiera actual y futura de la empresa así como de sus resultados, se debería observar un mejor ajuste en la regresión en la que se predice el futuro resultado a partir de datos actuales. Los resultados de este análisis de nuevo mantienen las conclusiones obtenidas en el análisis previo de relevancia valorativa, esto es, no parece existir suficiente evidencia que apoye una diferenciación entre las dos submuestras por lo que no podemos observar ninguna ventaja explicativa en los modelos al considerar el método de consolidación seleccionado para las empresas multigrupo.

Por último, nuestro estudio da un paso más en cuanto a las consecuencias en la elección del método de consolidación, y a partir de modelos de uso común en la literatura contable y financiera, se incorporan un cómputo de predicciones de resultados y valoración de acciones. Una vez estimados los futuros resultados y valores intrínsecos, se calculan los errores de predicción o valoración cometidos mediante comparación con los valores realmente 
observados. Obviamente, estos errores son consecuencia de la estimación de modelos en base a la información disponible, que habrá sido elaborada por las empresas partícipes en empresas multigrupo, aplicando los dos posibles criterios de consolidación.

Ambos análisis, tanto el de capacidad predictiva como el de capacidad valorativa de la información proporcionada, muestran resultados similares: las diferencias en los errores absolutos de predicción y valoración no son significativas, y de serlas la evidencia es ligeramente favorable en el subgrupo de empresa que ha optado por la puesta en equivalencia. Estos resultados se mantienen si tenemos en cuenta dos posibles factores endógenos que pueden estar afectando a los resultados obtenidos en nuestro estudio: el diferente tamaño y endeudamiento de las empresas de la muestra.

En definitiva, teniendo en cuenta las limitaciones en cuanto a la información financiera disponible de las empresas partícipes en empresas multigrupo en nuestro país y en base a los resultados obtenidos en este estudio, no parece que la utilización del método de integración proporcional para las empresas multigrupo proporcione información de más utilidad que la que se obtiene al utilizar como método la puesta en equivalencia. El establecimiento, por tanto, de un único método de consolidación para este tipo de empresas probablemente no conllevará una pérdida de información para los usuarios de la misma.

\section{REFERENCIAS BIBLIOGRÁFICAS}

AECA, 2005. Valoración de Pymes, Documentos AECA, Serie Valoración de Empresas, Documento № 7, Madrid.

Akaike, H., 1974. A new look at the statistical model identification. IEEE Transactions on Automatic Control 19 (6), 716-723.

ASB, 1997. FRS 9: Associates and Joint Ventures. UK: Accounting Standards Board.

Bauman, M., 2003. The impact and valuation of off-balance-sheet activities concealed by equity method accounting. Accounting Horizons, 17(4), 303-314.

Easton, P. y Sommers, G., 2003. Scale and the Scale Effect in Market-based Accounting Research. Journal of Business, Finance \& Accounting, 30, 25-56.

European Financial Reporting Advisory Group 2014. The equity method: a measurement basis or one-line consolidation?. EFRAG Short Discussion Series. January 2014. Disponible en: http://www.efrag.org/files/EFRAG\%20public\%20letters/EFRAG\%20SDS/SDS1 The Equity Met hod/EFRAG SDS1 The Equity Method.pdf

Fernández, P., 2004. Valoración de Empresas. Ediciones Deusto/Gestión 2000. 
Francis, J., Olsson P., y Oswald D.R., 2000. Comparing the Accuracy and Explainability of Dividend, Free Cash Flow, and Abnormal Earnings Equity Value Estimates". Journal of Accounting Research, 38 (1), 45-70.

García Ayuso, M., y Monterrey, J., 1998. El modelo de valoración Edwards-Bell-Ohlson (EBO): Aspectos teóricos y evidencia empírica. Revista Española de Financiación y Contabilidad, 96, 751-785.

García-Ayuso, M., Monterrey, J. y Pineda, C., 1999: Una evaluación empírica de los resultados anormales. Revista Española de Financiación y Contabilidad, 28 (98), 179-200.

Giner, B. y Verón, C.S., 2012. Determinantes de la discrecionalidad directiva en la elección del método contable de las participaciones en negocios conjuntos. Revista Española de Financiación y Contabilidad, 41 (156), 589-614.

Graham, R., King, R. y Morril, C., 2003. Decision usefulness of alternative joint venture reporting methods. Accounting Horizons, 17, 123-137.

$\mathrm{Gu}$, Z. (2007). "Across-sample Incomparability of $R^{2} s$ and Additional Evidence on Value Relevance Changes Over Time", Journal of Business, Finance \& Accounting, 34, 1073-1098

Hand, J., 2005. The value relevance of financial statements in the venture capital market. The Accounting Review, 80 (2), 613-648

Hand, J., y Landsman, W. R., 2005: The pricing of dividends in equity valuation. Journal of Business, Finance and Accounting, 32 (3\&4), 435-469

Holthausen, R. y Watts, R., 2001. The relevance of the value-relevance literature for financial accounting standard setting. Journal of Accounting and Economics, 31, 3-75.

Hennart, J., 1988. A transaction costs theory of equity joint ventures, Strategic Management Journal, 9(4), 361-374.

Kothavala, K., 2003: Proportional consolidation versus the equity method: a risk measurement perspective on reporting interests in joint ventures. Journal of Accounting and Public Policy, 22(6), 517-538.

Larrán, M. y Piñero, J. M., 2005. El modelo de Ohlson (1995): ¿Hemos llegado realmente a comprenderlo? Revista de Contabilidad (Spanish Accounting Review), 8 (16), 115-152.

Leung, H., 2011. Analysts Earnings Forecast Distribution. International Journal of Banking and Finance, 8(3), 37-53

Lourenço, I. y Dias, J., 2010. Determinants of the Accounting Choice Between Alternative Reporting Methods for Interests in Jointly Controlled Entities. European Accounting Review, 19 (4), 739-773

Makridakis, S., 1993. Accuracy measures: Theoretical and practical concerns. International Journal of Forecasting, 9(4), 527-529.

Marín, J. M. y Rubio, G., 2001. Economía Financiera. Antoni Bosch, editor S.A. Barcelona

Nobes, C. 2002. An Analysis of the International Development of the Equity Method. Abacus, 38: 16-45. doi: 10.1111/1467-6281.00096

O'Hanlon, J. y Taylor, P., 2007. The value relevance of disclosures of liabilities of equityaccounted investees: UK evidence. Accounting and Business Research, 37 (4), 267-284.

Ohlson, J. A., 1995. Earnings, Book Values, and Dividends in Equity Valuation. Contemporary Accounting Research, 11 (2), 661-687. 
Ohlson, J. A., 1999. On Transitory Earnings. Review of Accounting Studies, 4 (3 \& 4), 145-162.

Ohlson, J. A., 2001. Earnings, book values, and dividends in equity valuation: An empirical perspective. Contemporary Accounting Research, 18 (1), 107-120

Penman, S. y Sougiannis, T., 1998. A comparison of dividend, cash flow, and earnings approaches to equity valuation. Contemporary Accounting Research, 15 (3), 343-383.

Petersen, M.A., 2009. Estimating Standard Errors in Finance Panel Data Sets: Comparing Approaches. The Review of Financial Studies, 22 (1), 435-480.

Pricewaterhouse Coopers, 2003: Guía de valoración de empresas. Ed. Prentice Hall. Pearson Education. Coordinado por Miguel Sanjurjo y Mar Reinoso.

Rees, W. P., 1997. The impact of dividends, debt and investment on valuation models. Journal of Business, Finance and Accounting, 24 (7\&8), 1111-1140

Richardson, A.W., Roubi, R.R. y Soonawalla, K., 2012. Decline in Financial Reporting for Joint Ventures? Canadian Evidence on Removal of Financial Reporting Choice. European Accounting Review, 21 (2), 373-393.

Soonowalla, K., 2006. Accounting for Joint Ventures and Associates in Canada, UK, and US: Do US Rules Hide Information? Journal of Business Finance \& Accounting, 33(3 \& 4), 395-417.

Stoltzfus, R. y Epps, R., 2005. An empirical study of the value-relevance of using proportionate consolidation accounting for investments in joint ventures. Accounting Forum, 29, 169-190. 


\section{Tabla 1: Medias de las variables más relevantes. Total y por método de consolidación}

Columnas $\rightarrow \mathrm{N}$ : número de observaciones empresa-año de cada subgrupo con empresas multigrupo incluidas en sus cuentas consolidadas. Media: media de la variable indicada en cada fila para cada muestra; Submuestra IP: submuestra de observaciones empresa-año que ha aplicado el método de consolidación proporcional a sus participadas multigrupo; Submuestra PPE: submuestra de observaciones empresa-año que ha aplicado el procedimiento de puesta en equivalencia a sus participadas multigrupo; Test Dif. Medias: $p$-valor del contraste paramétrico de diferencia de medias entre la submuestra de consolidación proporcional y la de puesta en equivalencia.

Filas $\rightarrow$ ROA: rentabilidad sobre el activo; rai: resultado antes de impuestos; ta: total activo; ROE: rentabilidad sobre el patrimonio neto (rentabilidad financiera); pn: total patrimonio neto; REND1: ratio de endeudamiento medido como deuda/total activo; REND2: ratio de endeudamiento medido como deuda/patrimonio neto; deuda: total pasivo exigible; Rentdiv: rentabilidad por dividendos; ingr: total de ingresos; eps: beneficio por acción; precio: precio de cierre; capburs: capitalización bursátil al cierre;mb: ratio market to book; beta: beta o riesgo sistemático a fecha de cierre medido a través de rentabilidades de mercado de los 60 meses anteriores.

Las variables rai, ta, pn, deuda, ingr y capburs están medidas en millones de euros. Las variables eps y precio están medidas en Euros por Acción.

\begin{tabular}{|c|cc|cc|cc|c|}
\hline & Muestra & Completa & Submuestra & IP & Submuestra & PPE & Test \\
\hline Variable & $\mathbf{N}$ & Media & $\mathbf{N}$ & Media & $\mathbf{N}$ & Media & Dif. Medias \\
\hline ROA & 481 & $5,44 \%$ & 360 & $4,95 \%$ & 121 & $6,91 \%$ & 0,07 \\
rai & 488 & 730,13 & 367 & 766,33 & 121 & 620,33 & 0,42 \\
ta & 487 & 32746,47 & 366 & 31918,06 & 121 & 35252,23 & 0,78 \\
ROE & 488 & $5,84 \%$ & 367 & $0,66 \%$ & 121 & $21,55 \%$ & 0,00 \\
pn & 488 & 3316,07 & 367 & 3482,43 & 121 & 2811,48 & 0,35 \\
REND1 & 487 & 0,36 & 366 & 0,39 & 121 & 0,29 & 0,00 \\
REND2 & 488 & 3,39 & 367 & 3,64 & 121 & 2,65 & 0,04 \\
deuda & 488 & 11694,42 & 367 & 11657,47 & 121 & 11806,50 & 0,97 \\
Rentdiv & 487 & $2,61 \%$ & 367 & $2,27 \%$ & 120 & $3,65 \%$ & 0,00 \\
ingr & 487 & 5308,65 & 366 & 5840,59 & 121 & 3699,64 & 0,02 \\
eps & 478 & 1,16 & 358 & 1,22 & 120 & 0,99 & 0,22 \\
precio & 488 & 16,34 & 367 & 16,99 & 121 & 14,38 & 0,17 \\
capburs & 488 & 6525,60 & 367 & 6937,50 & 121 & 5276,28 & 0,21 \\
mb & 488 & 2,90 & 367 & 2,78 & 121 & 3,28 & 0,18 \\
beta & 464 & 0,92 & 345 & 0,93 & 119 & 0,87 & 0,27 \\
\hline
\end{tabular}


Tabla 2: Medianas de las variables más relevantes. Total y por método de consolidación

Columnas $\rightarrow \mathrm{N}$ : número de observaciones empresa-año de cada subgrupo con empresas multigrupo incluidas en sus cuentas consolidadas; Mediana: mediana de la variable indicada en cada fila para cada muestra; Submuestra IP: submuestra de observaciones empresa-año que ha aplicado el método de consolidación proporcional a sus participadas multigrupo; Submuestra PPE: submuestra de observaciones empresa-año que ha aplicado el procedimiento de puesta en equivalencia a sus participadas multigrupo; Test Dif. Medianas: p-valor del contraste no paramétrico de diferencia de medianas entre la submuestra de consolidación proporcional y la de puesta en equivalencia

Filas $\rightarrow$ ROA: rentabilidad sobre el activo; rai: resultado antes de impuestos; ta: total activo; ROE: rentabilidad sobre el patrimonio neto (rentabilidad financiera); pn: total patrimonio neto; REND1: ratio de endeudamiento medido como deuda/total activo; REND2: ratio de endeudamiento medido como deuda/patrimonio neto; deuda: total pasivo exigible; Rentdiv: rentabilidad por dividendos; ingr: total de ingresos; eps: beneficio por acción; precio: precio de cierre; capburs: capitalización bursátil al cierre; mb: ratio market to book; beta: beta o riesgo sistemático a fecha de cierre medido a través de rentabilidades de mercado de los 60 meses anteriores.

Las variables rai, ta, pn, deuda, ingr y capburs están medidas en millones de euros. Las variables eps y precio están medidas en Euros por Acción.

\begin{tabular}{|c|cc|cc|cc|c|}
\hline & Muestra & Completa & Submuestra & IP & Submuestra & PPE & Test \\
\hline Variable & $\mathbf{N}$ & Mediana & $\mathbf{N}$ & Mediana & $\mathbf{N}$ & Mediana & $\begin{array}{c}\text { Dif. } \\
\text { Medianas }\end{array}$ \\
\hline ROA & 481 & $5,08 \%$ & 360 & $5,17 \%$ & 121 & $4,86 \%$ & 0,48 \\
rai & 488 & 107,19 & 367 & 111,03 & 121 & 103,05 & 0,46 \\
ta & 487 & 3225,62 & 366 & 3805,01 & 121 & 2343,61 & 0,03 \\
ROE & 488 & $16,81 \%$ & 367 & $16,99 \%$ & 121 & $15,93 \%$ & 0,75 \\
pn & 488 & 616,72 & 367 & 628,72 & 121 & 616,02 & 0,75 \\
REND1 & 487 & 0,35 & 366 & 0,38 & 121 & 0,29 & 0,00 \\
REND2 & 488 & 1,52 & 367 & 1,72 & 121 & 1,12 & 0,07 \\
deuda & 488 & 1092,89 & 367 & 1339,06 & 121 & 633,34 & 0,12 \\
rentdiv & 487 & $1,79 \%$ & 367 & $1,52 \%$ & 120 & $2,87 \%$ & 0,00 \\
ingr & 487 & 1099,84 & 366 & 1141,06 & 121 & 979,01 & 0,36 \\
eps & 478 & 0,62 & 358 & 0,69 & 120 & 0,56 & 0,14 \\
precio & 488 & 10,28 & 367 & 10,48 & 121 & 9,80 & 0,60 \\
capburs & 488 & 1692,16 & 367 & 1669,35 & 121 & 1714,98 & 0,92 \\
bm & 488 & 1,98 & 367 & 1,99 & 121 & 1,96 & 0,92 \\
beta & 464 & 0,88 & 345 & 0,89 & 119 & 0,85 & 0,46 \\
\hline
\end{tabular}


Tabla 3: Análisis descriptivo de series temporales

La siguiente tabla muestra los coeficientes estimados individualmente para cada empresa, en serie temporal. Un modelo AR (1) se basa en la siguiente regresión para cada empresa por separado: $x_{i t}=\propto_{0}+\propto_{1} \cdot x_{i t-1}+e_{t}$ Un modelo AR (2) en la siguiente regresión: $x_{i t}=\propto_{0}+\propto_{1} \cdot x_{i t-1}+\propto_{2} \cdot x_{i t-2}+e_{t}$

$Y$ un modelo de tendencia temporal (Ten. Temp): $x_{i t}=\propto_{0}+\propto_{1} \cdot t+e_{t}$

Donde $\mathrm{x}$ : es la variable a analizar en serie temporal, y $\mathrm{t}$ es el año concreto en el que se observa dicha variable.

Variable: variable utilizada en la regresión en serie temporal; tipo: tipo de modelo utilizado; Media: media del coeficiente individual y del $\mathrm{R}^{2}$ estimado en cada regresión para cada empresa; Mediana: media del coeficiente individual y del $\mathrm{R}^{2}$ estimado en cada regresión para cada empresa; \%sign: porcentaje de empresas en las que la variable estimada es significativa al $10 \%$;

Panel A: Toda la muestra

\begin{tabular}{|c|c|c|c|c|c|c|c|c|c|}
\hline Variable & Tipo & $\begin{array}{c}\text { Media } \\
\alpha_{1}\end{array}$ & $\alpha_{2}$ & $\begin{array}{c}\text { Mediana } \\
\alpha_{1}\end{array}$ & $\alpha_{2}$ & $\begin{array}{c}\% \text { sign } \\
\alpha_{1}\end{array}$ & $\begin{array}{c}\text { \%sign } \\
\alpha_{2}\end{array}$ & \begin{tabular}{|c|} 
Media \\
$\mathrm{R}^{2}$
\end{tabular} & $\begin{array}{c}\text { Mediana } \\
\mathrm{R}^{2}\end{array}$ \\
\hline \multirow{3}{*}{$\begin{array}{l}\text { Resultado } \\
\text { (millones } € \text { ) }\end{array}$} & $A R(1)$ & 0,75 & & 0,77 & & $76,25 \%$ & & 0,54 & 0,56 \\
\hline & $A R(2)$ & 0,94 & $-0,36$ & 0,83 & $-0,14$ & $68,83 \%$ & $24,68 \%$ & 0,63 & 0,64 \\
\hline & Ten. Temp. & 71,53 & & 8,42 & & $51,25 \%$ & & 0,41 & 0,31 \\
\hline Resultado & AR (1) & 0,56 & & 0,57 & & $61,04 \%$ & & 0,47 & 0,46 \\
\hline deflactado & $A R(2)$ & 0,42 & 0,16 & 0,37 & 0,17 & $36,36 \%$ & $21,21 \%$ & 0,52 & 0,61 \\
\hline cap. bursátil & Ten. Temp. & $-0,03$ & & 0,00 & & $38,96 \%$ & & 0,33 & 0,30 \\
\hline
\end{tabular}

Panel B: Muestra que ha optado por la integración proporcional

\begin{tabular}{|c|c|c|c|c|c|c|c|c|c|}
\hline Variable & Tipo & $\begin{array}{c}\text { Media } \\
\alpha_{1}\end{array}$ & $\alpha_{2}$ & $\begin{array}{c}\text { Mediana } \\
\alpha_{1}\end{array}$ & $\alpha_{2}$ & $\begin{array}{c}\% \text { sign } \\
\alpha_{1}\end{array}$ & $\begin{array}{c}\text { \%sign } \\
\alpha_{2}\end{array}$ & $\begin{array}{c}\text { Media } \\
\mathrm{R}^{2}\end{array}$ & $\begin{array}{c}\text { Mediana } \\
\mathbf{R}^{\mathbf{2}}\end{array}$ \\
\hline \multirow{3}{*}{$\begin{array}{l}\text { Resultado } \\
\text { (millones } € \text { ) }\end{array}$} & AR (1) & 0,79 & \multirow{3}{*}{$-0,43$} & 0,75 & \multirow{3}{*}{$-0,14$} & $73,21 \%$ & \multirow{3}{*}{$22,64 \%$} & 0,53 & 0,53 \\
\hline & AR (2) & 1,01 & & 0,77 & & $67,92 \%$ & & 0,63 & 0,63 \\
\hline & Ten. Temp. & 84,03 & & 5,84 & & $53,57 \%$ & & 0,45 & 0,35 \\
\hline Resultado & AR (1) & 0,56 & \multirow{3}{*}{0,10} & 0,53 & \multirow{3}{*}{0,17} & $55,56 \%$ & \multirow{3}{*}{$23,26 \%$} & 0,44 & 0,39 \\
\hline deflactado & AR (2) & 0,47 & & 0,40 & & $39,53 \%$ & & 0,50 & 0,56 \\
\hline cap. bursátil & Ten. Temp. & $-0,04$ & & $-0,01$ & & $42,59 \%$ & & 0,35 & 0,36 \\
\hline
\end{tabular}

Panel C: Muestra que ha optado por la puesta en equivalencia

\begin{tabular}{|c|c|c|c|c|c|c|c|c|c|}
\hline Variable & Tipo & $\begin{array}{c}\text { Media } \\
\alpha_{1}\end{array}$ & $\alpha_{2}$ & $\begin{array}{c}\text { Mediana } \\
\alpha_{1}\end{array}$ & $\alpha_{2}$ & $\begin{array}{c}\% \text { sign } \\
\alpha_{1}\end{array}$ & $\begin{array}{c}\text { \%sign } \\
\alpha_{2}\end{array}$ & $\begin{array}{c}\text { Media } \\
\mathrm{R}^{2}\end{array}$ & $\begin{array}{c}\text { Mediana } \\
\mathbf{R}^{\mathbf{2}}\end{array}$ \\
\hline \multirow{3}{*}{$\begin{array}{c}\text { Resultado } \\
\text { (millones } € \text { ) }\end{array}$} & AR (1) & 0,66 & \multirow{3}{*}{$-0,19$} & 0,79 & \multirow{3}{*}{$-0,12$} & $83,33 \%$ & \multirow{3}{*}{$29,17 \%$} & 0,57 & 0,62 \\
\hline & $A R(2)$ & 0,80 & & 0,84 & & $70,83 \%$ & & 0,64 & 0,68 \\
\hline & Ten. Temp. & 42,35 & & 10,68 & & $45,83 \%$ & & 0,33 & 0,23 \\
\hline Resultado & AR (1) & 0,56 & \multirow{3}{*}{0,28} & 0,73 & \multirow{3}{*}{0,21} & $73,91 \%$ & \multirow{3}{*}{$17,39 \%$} & 0,54 & 0,62 \\
\hline deflactado & $A R(2)$ & 0,32 & & 0,34 & & $30,43 \%$ & & 0,57 & 0,64 \\
\hline cap. bursátil & Ten. Temp. & $-0,01$ & & 0,00 & & $30,43 \%$ & & 0,29 & 0,21 \\
\hline
\end{tabular}


Tabla 4: Coeficientes de relevancia valorativa. Total y por método de consolidación.

La siguiente tabla muestra los resultados de las regresiones de relevancia valorativa [1] y [2]:

$P_{i t}=\alpha_{1}+\alpha_{2} b v_{i t}+\alpha_{3} x_{i t}+\varepsilon_{i t}$

$P_{i t}=\alpha_{1}+\alpha_{11} P P E_{i t}+\alpha_{2} b v_{i t}+\alpha_{21} b v_{i t} P P E_{i t}+\alpha_{3} x_{i t}+\alpha_{31} x_{i t} P P E_{i t}+\varepsilon_{i t}$

Donde P: precio de mercado, bv: patrimonio neto; $\mathrm{x}$ : resultado del ejercicio; PPE: variable dummy que toma el valor 1 si la empresa ha optado por el procedimiento de puesta en equivalencia.

La estimación se realiza mediante tres procedimientos: deflactando por la capitalización bursátil a inicio del periodo, transformación logarítmica, y datos de panel (efectos fijos) con variables no deflactadas. La significatividad de cada coeficiente se mide a través del $p$-valor del contraste bilateral de significatividad de cada coeficiente utilizando la estimación por clusters de Petersen (2009). En el caso de datos de panel no mostramos el valor del intercepto, ya que con esta técnica de estimación hay tantos interceptos como empresas en la muestra.

IP: submuestra de observaciones empresa-año que ha aplicado el método de consolidación proporcional a sus participadas multigrupo; PPE: submuestra de observaciones empresa-año que ha aplicado el procedimiento de puesta en equivalencia a sus participadas multigrupo; $\mathrm{N}$ : número de observaciones total de cada regresión; $\mathrm{R}^{2}$ : coeficiente de determinación $\mathrm{R}^{2}$ ajustado, AIC: Criterio de la información de Akaike.

Panel A: Estimación con datos deflactados por la capitalización bursátil a inicio del periodo

\begin{tabular}{|c|c|cccccc|ccc|}
\hline Ecuación & Muestra & $\boldsymbol{\alpha}_{1}$ & $\boldsymbol{\alpha}_{11}$ & $\boldsymbol{\alpha}_{2}$ & $\boldsymbol{\alpha}_{21}$ & $\boldsymbol{\alpha}_{3}$ & $\boldsymbol{\alpha}_{31}$ & $\mathbf{N}$ & $\mathbf{R}^{2}$ & AIC \\
\hline$[1]$ & Toda & $0,79^{* * *}$ & & $0,39^{* * *}$ & & $0,92^{* * *}$ & & 475 & 0,22 & 1,54 \\
& IP & $0,79^{* * *}$ & & $0,41^{* * *}$ & & $0,80^{* * *}$ & & 356 & 0,22 & 1,60 \\
& PPE & $0,79^{* * *}$ & & $0,24^{*}$ & & $1,83^{* * *}$ & & 119 & 0,24 & 1,35 \\
\hline [2] & Toda & $0,79^{* * *}$ & $-0,00$ & $0,41^{* * *}$ & $-0,17$ & $0,80^{* * *}$ & $1,03^{*}$ & 475 & 0,22 & 1,55 \\
\hline
\end{tabular}

*** Estadísticamente significativo al $1 \% ; * *$ al $5 \% ; *$ al $10 \%$

Panel B: Estimación por transformación logarítmica

\begin{tabular}{|c|c|cccccc|ccc|}
\hline Ecuación & Muestra & $\boldsymbol{\alpha}_{1}$ & $\boldsymbol{\alpha}_{11}$ & $\boldsymbol{\alpha}_{2}$ & $\boldsymbol{\alpha}_{21}$ & $\boldsymbol{\alpha}_{3}$ & $\boldsymbol{\alpha}_{31}$ & $\mathbf{N}$ & $\mathbf{R}^{\mathbf{2}}$ & AIC \\
\hline [1] & Toda & $1,58^{* * *}$ & & $0,66^{* * *}$ & & $0,26^{* * *}$ & & 488 & 0,76 & 2,73 \\
& IP & $1,37^{* * *}$ & & $0,70^{* * *}$ & & $0,24^{* * *}$ & & 367 & 0,77 & 2,80 \\
& PPE & $2,36^{* * *}$ & & $0,51^{* * *}$ & & $0,34^{* * *}$ & & 121 & 0,74 & 2,42 \\
\hline$[2]$ & Toda & $1,37^{* * *}$ & $0,98^{* *}$ & $0,70^{* * *}$ & $-0,19^{* *}$ & $0,24^{* *}$ & $-0,10$ & 488 & 0,76 & 2,72 \\
\hline
\end{tabular}

*** Estadísticamente significativo al $1 \% ; * *$ al $5 \% ; *$ al $10 \%$

Panel C: Datos de panel: modelo efectos fijos

\begin{tabular}{|c|c|c|c|c|c|c|c|c|c|c|}
\hline Ecuación & Muestra & $\alpha_{1}$ & $\alpha_{11}$ & $\alpha_{2}$ & $\alpha_{21}$ & $\alpha_{3}$ & $\alpha_{31}$ & $\mathbf{N}$ & $\mathbf{R}^{2}$ & AIC \\
\hline \multirow[t]{3}{*}{ [1] } & Toda & & & $0,64^{* * *}$ & & $1,10^{* * *}$ & & 488 & 0,33 & 1,27 \\
\hline & IP & & & $0,71^{* * *}$ & & $0,99^{* * *}$ & & 367 & 0,35 & 1,29 \\
\hline & PPE & & & $0,42^{* * *}$ & & $1,73^{* * *}$ & & 121 & 0,31 & 1,06 \\
\hline [2] & Toda & & & $0,72^{* * *}$ & $-0,34^{* *}$ & $1,00^{* * *}$ & 0,91 & 488 & 0,34 & 1,25 \\
\hline
\end{tabular}

*** Estadísticamente significativo al 1\%; ** al 5\%; * al $10 \%$ 
Tabla 5: Coeficientes modelo de predicción. Total y por método de consolidación.

La siguiente tabla muestra los resultados de las regresiones de capacidad predictiva [3] y [4]:

$x_{i t+1}=\alpha_{1}+\alpha_{2} b v_{i t}+\alpha_{3} x_{i t}+\varepsilon_{i t}$

$x_{i t+1}=\alpha_{1}+\alpha_{11} P P E_{i t}+\alpha_{2} b v_{i t}+\alpha_{21} b v_{i t} P P E_{i t}+\alpha_{3} x_{i t}+\alpha_{31} x_{i t} P P E_{i t}+\varepsilon_{i t}$

Donde P: precio de mercado, bv: patrimonio neto; $\mathrm{x}$ : resultado del ejercicio; PPE: variable dummy que toma el valor 1 si la empresa ha optado por el procedimiento de puesta en equivalencia.

La estimación se realiza mediante tres procedimientos: deflactando por la capitalización bursátil a inicio del periodo, transformación logarítmica, y datos de panel (efectos fijos) con variables no deflactadas. La significatividad de cada coeficiente se mide a través del $p$-valor del contraste bilateral de significatividad de cada coeficiente utilizando la estimación por clusters de Petersen (2009). En el caso de datos de panel no mostramos el valor del intercepto, ya que con esta técnica de estimación hay tantos interceptos como empresas en la muestra.

IP: submuestra de observaciones empresa-año que ha aplicado el método de consolidación proporcional a sus participadas multigrupo; PPE: submuestra de observaciones empresa-año que ha aplicado el procedimiento de puesta en equivalencia a sus participadas multigrupo; $\mathrm{N}$ : número de observaciones total de cada regresión; $\mathrm{R}^{2}$ : coeficiente de determinación $\mathrm{R}^{2}$ ajustado, AIC: Criterio de la información de Akaike.

Panel A: Estimación con datos deflactados por la capitalización bursátil a inicio del periodo

\begin{tabular}{|c|c|cccccc|ccc|}
\hline Ecuación & Muestra & $\boldsymbol{\alpha}_{1}$ & $\boldsymbol{\alpha}_{11}$ & $\boldsymbol{\alpha}_{2}$ & $\boldsymbol{\alpha}_{21}$ & $\boldsymbol{\alpha}_{3}$ & $\boldsymbol{\alpha}_{31}$ & $\mathbf{N}$ & $\mathbf{R}^{\mathbf{2}}$ & AIC \\
\hline [3] & Toda & $0,02^{*}$ & & $-0,03^{*}$ & & $0,67^{* * *}$ & & 383 & 0,39 & 1,58 \\
& IP & 0,02 & & $-0,03^{*}$ & & $0,68^{* * *}$ & & 289 & 0,40 & 1,49 \\
& PPE & 0,03 & & $-0,01$ & & $0,58^{* * *}$ & & 94 & 0,32 & 1,87 \\
\hline [4] & Toda & 0,02 & 0,008 & $-0,03^{*}$ & 0,024 & $0,68^{* * *}$ & $-0,11$ & 383 & 0,39 & 1,57 \\
\hline
\end{tabular}

*** Estadísticamente significativo al $1 \% ;{ }^{* *}$ al $5 \% ; *$ al $10 \%$

Panel B: Estimación por transformación logarítmica

\begin{tabular}{|c|c|cccccc|ccc|}
\hline Ecuación & Muestra & $\boldsymbol{\alpha}_{1}$ & $\boldsymbol{\alpha}_{11}$ & $\boldsymbol{\alpha}_{\mathbf{2}}$ & $\boldsymbol{\alpha}_{21}$ & $\boldsymbol{\alpha}_{3}$ & $\boldsymbol{\alpha}_{31}$ & $\mathbf{N}$ & $\mathbf{R}^{2}$ & AIC \\
\hline [3] & Toda & $-0,70^{* * *}$ & & $0,45^{* * *}$ & & $0,54^{* * *}$ & & 409 & 0,76 & 2,92 \\
& IP & $-0,73^{* * *}$ & & $0,47^{* * *}$ & & $0,53^{* * *}$ & & 312 & 0,78 & 2,93 \\
& PPE & $-0,56$ & & $0,36^{* * *}$ & & $0,61^{* * *}$ & & 97 & 0,70 & 2,95 \\
\hline [4] & Toda & $-0,73^{* * *}$ & 0,17 & $0,47^{* * *}$ & $-0,11$ & $0,53^{* * *}$ & 0,08 & 409 & 0,76 & 2,93 \\
\hline
\end{tabular}

*** Estadísticamente significativo al $1 \% ; * *$ al $5 \% ; *$ al $10 \%$

Panel C: Datos de panel (efectos fijos)

\begin{tabular}{|c|c|c|c|c|c|c|c|c|c|c|}
\hline Ecuación & Muestra & $\alpha_{1}$ & $\alpha_{11}$ & $\alpha_{2}$ & $\alpha_{21}$ & $\alpha_{3}$ & $\alpha_{31}$ & $\mathbf{N}$ & $\mathbf{R}^{2}$ & AIC \\
\hline \multirow[t]{3}{*}{ [3] } & Toda & & & $0,04^{* * *}$ & & $0,21^{* * *}$ & & 409 & 0,12 & 2,87 \\
\hline & IP & & & $0,04^{* * *}$ & & $0,24^{* * *}$ & & 312 & 0,13 & 2,95 \\
\hline & PPE & & & 0,03 & & 0,19 & & 97 & 0,15 & 2,29 \\
\hline [4] & Toda & & & $0,04^{* * *}$ & 0,02 & $0,25^{* * *}$ & $-0,26^{*}$ & 409 & 0,13 & 2,87 \\
\hline
\end{tabular}

*** Estadísticamente significativo al 1\%; ** al 5\%; * al 10\% 
Tabla 6: Errores de predicción. Total y por método de consolidación.

La siguiente tabla muestra los errores absolutos de predicción de las estimaciones de beneficios a 1 año, realizadas utilizando los modelos [P1] a [P6]. [P1] es un modelo de recorrido aleatorio, [P2] un modelo con tendencia temporal, [P3] un modelo $A R(1),[P 4]$ un modelo $A R(2),[P 5]$ un modelo $A R(1)$ con tendencia temporal, $[P 6]$ un modelo $A R(1)$ con patrimonio neto como variable relevante.

IP: submuestra de observaciones empresa-año que ha aplicado el método de consolidación proporcional a sus participadas multigrupo; PPE: submuestra de observaciones empresa-año que ha aplicado el procedimiento de puesta en equivalencia a sus participadas multigrupo. P-V test: $p$-valor del contraste de diferencia en los errores absolutos de predicción entre las submuestras de integración proporcional y puesta en equivalencia, siendo la hipótesis nula la de igualdad de errores medianos en cada par de submuestras IP y PPE.

Se muestra el error absoluto para cada submuestra (integración proporcional y puesta en equivalencia) por separado. Concretamente, para los modelos [P2] a [P6] se muestra el error absoluto mediano de toda la muestra y de cada submuestra de empresas según si los coeficientes de cada modelo se han estimado en sección cruzada o en serie temporal. En el caso de sección cruzada si se han estimado los mismos coeficientes para todas las empresas (global de toda la muestra), o si se han estimado para cada submuestra (integración proporcional vs. puesta en equivalencia).

Panel A: Modelo [P1] -no requiere estimaciones de coeficientes de predicción-

\begin{tabular}{ccc}
\hline Modelo & Muestra & $\begin{array}{c}\text { Mediana del error absoluto de } \\
\text { predicción a } \mathbf{1} \text { año }\end{array}$ \\
\hline \multirow{3}{*}{ [P1] } & Toda & 0,0275 \\
& IP & 0,0269 \\
& PPE & 0,0288 \\
& P-V difer & 0,8081 \\
\hline
\end{tabular}

Panel B: Modelo [P2] a [P6] -requieren estimaciones de coeficientes de predicción-

\begin{tabular}{|c|c|c|c|c|}
\hline Modelo & Muestra & $\begin{array}{c}\text { Mediana del error } \\
\text { absoluto de predicción } \\
\text { a } 1 \text { año } \\
\text { (sección cruzada global } \\
\text { para toda la muestra) }\end{array}$ & $\begin{array}{l}\text { Mediana del error } \\
\text { absoluto de } \\
\text { predicción a } 1 \text { año } \\
\text { (sección cruzada por } \\
\text { submuestras) } \\
\end{array}$ & $\begin{array}{l}\text { Mediana del error } \\
\text { absoluto de } \\
\text { predicción a } 1 \text { año } \\
\text { (serie temporal) }\end{array}$ \\
\hline & Toda & 0,0601 & 0,0598 & 0,0313 \\
\hline \multirow[t]{4}{*}{ [P2] } & IP & 0,0666 & 0,0687 & 0,0334 \\
\hline & PPE & 0,0462 & 0,0425 & 0,0243 \\
\hline & P-V difer & 0,0464 & 0,0071 & 0,0339 \\
\hline & Toda & 0,0397 & 0,0391 & 0,0271 \\
\hline \multirow[t]{4}{*}{ [P3] } & IP & 0,0407 & 0,0409 & 0,0271 \\
\hline & PPE & 0,0349 & 0,0325 & 0,0273 \\
\hline & P-V difer & 0,4970 & 0,0359 & 0,9777 \\
\hline & Toda & 0,0380 & 0,0380 & 0,0232 \\
\hline \multirow[t]{4}{*}{ [P4] } & IP & 0,0396 & 0,0396 & 0,0226 \\
\hline & PPE & 0,0349 & 0,0321 & 0,0236 \\
\hline & P-V difer & 0,2915 & 0,1242 & 0,8022 \\
\hline & Toda & 0,0369 & 0,0375 & 0,0278 \\
\hline \multirow[t]{4}{*}{ [P5] } & IP & 0,0390 & 0,0384 & 0,0308 \\
\hline & PPE & 0,0343 & 0,0306 & 0,0226 \\
\hline & P-V difers & 0,4970 & 0,1650 & 0,2019 \\
\hline & Toda & 0,0383 & 0,0381 & 0,0211 \\
\hline \multirow[t]{3}{*}{ [P6] } & IP & 0,0403 & 0,0401 & 0,0199 \\
\hline & PPE & 0,0338 & 0,0310 & 0,0232 \\
\hline & P-V difers & 0,2493 & 0,1042 & 0,3695 \\
\hline
\end{tabular}


Tabla 7: Errores de valoración. Total y por método de consolidación.

La siguiente tabla muestra los errores absolutos de valoración de las estimaciones de valor intrínseco, realizadas utilizando los modelos [V1] a [V4]. [V1] es un modelo de valoración en base al balance, [V2] un modelo de valoración basado en el resultado, [V3] un modelo de valoración basado en el resultado residual o anormal, [V4] un modelo de valoración basado en una regresión del precio histórico sobre las dos variables contables fundamentales, resultado y patrimonio, y [V5] es un modelo de valoración basado en Ohlson (2001).

IP: submuestra de observaciones empresa-año que ha aplicado el método de consolidación proporcional a sus participadas multigrupo; PPE: submuestra de observaciones empresa-año que ha aplicado el procedimiento de puesta en equivalencia a sus participadas multigrupo. P-V test: $p$-valor del contraste de diferencia en los errores absolutos de valoración entre las submuestras de integración proporcional y puesta en equivalencia, siendo la hipótesis nula la de igualdad de errores medianos en cada par de submuestras IP y PPE.

Se muestra el error absoluto para cada submuestra (integración proporcional y puesta en equivalencia) por separado. Concretamente, para los modelos [V4] y [V5] se muestra el error absoluto mediano de toda la muestra y de cada submuestra de empresas según si los coeficientes de cada modelo se han estimado en sección cruzada o en serie temporal. En el caso de sección cruzada si se han estimado los mismos coeficientes para todas las empresas (global de toda la muestra), o si se han estimado para cada submuestra (integración proporcional vs. puesta en equivalencia).

Panel A: Modelos [V1] a [V3] -no requieren estimaciones de coeficientes de valoración-

\begin{tabular}{ccc}
\hline Modelo & Muestra & $\begin{array}{c}\text { Mediana del error absoluto } \\
\text { de valoración }\end{array}$ \\
\hline [V1] & Toda & 0,5666 \\
& IP & 0,5730 \\
& PPE & 0,5262 \\
\hline \multirow{2}{*}{ V2] } & P-V difer & 0,4748 \\
& Toda & 0,5391 \\
& IP & 0,5812 \\
& PPE & 0,4293 \\
[V3] & P-V difer & 0,0160 \\
& Toda & 0,5754 \\
& IP & 0,6056 \\
& PPE & 0,5432 \\
& P-V difer & 0,2942 \\
\hline
\end{tabular}

Panel B: Modelos [V4] a [V5] - requieren estimaciones de coeficientes de valoración-

\begin{tabular}{ccccc}
\hline Modelo & Muestra & $\begin{array}{c}\text { Mediana del error } \\
\text { absoluto de valoración } \\
\text { (sección cruzada global } \\
\text { para toda la muestra) }\end{array}$ & $\begin{array}{c}\text { Mediana del error } \\
\text { absoluto de valoración } \\
\text { (sección cruzada por } \\
\text { submuestras) }\end{array}$ & $\begin{array}{c}\text { Mediana del error } \\
\text { absoluto de } \\
\text { valoración } \\
\text { (serie temporal) }\end{array}$ \\
\hline \multirow{2}{*}{ [V4] } & Toda & 0,3186 & 0,3260 & 0,3180 \\
& IP & 0,3224 & 0,3324 & 0,3190 \\
& PPE & 0,3001 & 0,3201 & 0,3172 \\
& P-V difer & 0,8365 & 0,6579 & 0,9347 \\
\hline \multirow{2}{*}{ [V5] } & Toda & 0,2999 & 0,2935 & 0,2715 \\
& IP & 0,2992 & 0,2951 & 0,2706 \\
& PPE & 0,3005 & 0,2799 & 0,2785 \\
& P-V difer & 0,8858 & 0,6666 & 0,8441 \\
\hline
\end{tabular}


Tabla 8: Errores de predicción por tamaño, endeudamiento y método de consolidación.

La siguiente tabla muestra la diferencia entre la mediana del error absoluto de predicción de la muestra de empresas que ha optado por la integración proporcional y la que ha optado por la puesta en equivalencia, distinguiendo en cada subgrupo las empresas por tamaño y endeudamiento. Deuda: cociente entre la Deuda y el Total Activo; Tamaño: Total Activo

IP-PPE: diferencia entre la mediana del error absoluto de predicción de la muestra de empresas que ha optado por la integración proporcional y la que ha optado por la puesta en equivalencia. Si la diferencia es positiva, el error mediano es superior en las empresas IP, si la diferencia es negativa el error es mayor en las empresas PPE.

+ Deuda: submuestra de empresas más endeudadas; - Deuda: submuestra de empresas menos endeudada; + Tamaño: submuestra de empresas más grandes; - Tamaño: submuestra de empresas más pequeña.

Panel A: Modelo [P1] -no requiere estimaciones de coeficientes de predicción-

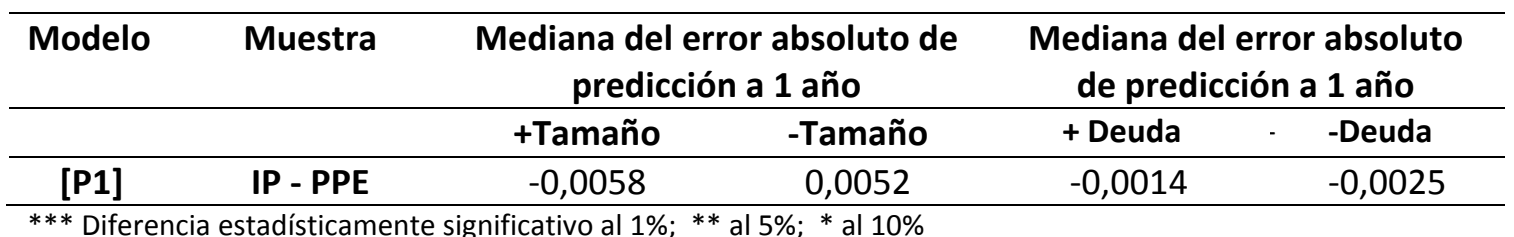

Panel B: Modelo [P2] a [P6] -requieren estimaciones de coeficientes de predicción-

\begin{tabular}{|c|c|c|c|c|c|c|c|}
\hline \multirow[t]{2}{*}{ Modelo } & \multirow[t]{2}{*}{ Muestra } & \multicolumn{2}{|c|}{$\begin{array}{c}\text { Mediana del error } \\
\text { absoluto de predicción } \\
\text { a } 1 \text { año } \\
\text { (sección cruzada global } \\
\text { para toda la muestra) }\end{array}$} & \multicolumn{2}{|c|}{$\begin{array}{l}\text { Mediana del error } \\
\text { absoluto de } \\
\text { predicción a } 1 \text { año } \\
\text { (sección cruzada por } \\
\text { submuestras) }\end{array}$} & \multicolumn{2}{|c|}{$\begin{array}{l}\text { Mediana del error } \\
\text { absoluto de } \\
\text { predicción a } 1 \text { año } \\
\text { (serie temporal) }\end{array}$} \\
\hline & & +Tamaño & -Tamaño & +Tamaño & -Tamaño & +Tamaño & -Tamaño \\
\hline [P2] & IP-PPE & 0,0205 & $0,0154^{*}$ & $0,0248^{*}$ & 0,0288 & 0,0011 & $0,0193^{* *}$ \\
\hline [P3] & IP-PPE & 0,0057 & 0,0056 & $0,0103^{* *}$ & 0,0134 & $-0,0043$ & 0,0049 \\
\hline [P4] & IP-PPE & $0,0067^{*}$ & $-0,0031$ & $0,0106^{* *}$ & 0,0010 & 0,0000 & $-0,0007$ \\
\hline [P5] & IP-PPE & 0,0039 & 0,0037 & $0,0088^{*}$ & 0,0048 & 0,0043 & 0,0112 \\
\hline \multirow[t]{2}{*}{ [P6] } & IP-PPE & 0,0063 & 0,0063 & 0,0081 & 0,0068 & $-0,0024$ & $-0,0032$ \\
\hline & & + Deuda & -Deuda & + Deuda & -Deuda & + Deuda & -Deuda \\
\hline [P2] & IP-PPE & $0,0515^{* * *}$ & 0,0000 & $0,0507^{* * *}$ & 0,0115 & $0,0166^{*}$ & 0,0033 \\
\hline [P3] & IP-PPE & $0,0194^{* * *}$ & $-0,0102$ & $0,0246^{* * *}$ & 0,0032 & $-0,0007$ & $-0,0010$ \\
\hline [P4] & IP-PPE & $0,0188^{* *}$ & $-0,0091$ & $0,0218^{* * *}$ & $-0,0036$ & $-0,0028$ & $-0,0022$ \\
\hline [P5] & IP-PPE & $0,0140^{* *}$ & $-0,0076$ & $0,0216^{* * *}$ & $-0,0011$ & $0,0183^{* *}$ & $-0,0004$ \\
\hline [P6] & IP-PPE & $0,0138^{* * *}$ & 0,0013 & $0,0183^{* * *}$ & 0,0046 & $-0,0039$ & $-0,0018$ \\
\hline
\end{tabular}

*** Diferencia estadísticamente significativo al $1 \% ;{ }^{* *}$ al $5 \% ;{ }^{*}$ al $10 \%$ 
Tabla 9: Errores de valoración por tamaño, endeudamiento y método de consolidación.

La siguiente tabla muestra la diferencia entre la mediana del error absoluto de valoración de la muestra de empresas que ha optado por la integración proporcional y la que ha optado por la puesta en equivalencia, distinguiendo en cada subgrupo las empresas por tamaño y endeudamiento. Deuda: cociente entre la Deuda y el Total Activo; Tamaño: Total Activo

IP-PPE: diferencia entre la mediana del error absoluto de predicción de la muestra de empresas que ha optado por la integración proporcional y la que ha optado por la puesta en equivalencia. Si la diferencia es positiva, el error mediano es superior en las empresas IP, si la diferencia es negativa el error es mayor en las empresas PPE.

+ Deuda: submuestra de empresas más endeudadas; - Deuda: submuestra de empresas menos endeudada; + Tamaño: submuestra de empresas más grandes; - Tamaño: submuestra de empresas más pequeña.

Panel A: Modelos [V1] a [V3] -no requieren estimaciones de coeficientes de valoración-

\begin{tabular}{cccccc}
\hline Modelo & Muestra & \multicolumn{2}{c}{$\begin{array}{c}\text { Mediana del error absoluto de } \\
\text { predicción a 1 año }\end{array}$} & \multicolumn{2}{c}{$\begin{array}{c}\text { Mediana del error absoluto } \\
\text { de predicción a } 1 \text { año }\end{array}$} \\
\hline & & +Tamaño & -Tamaño & + Deuda & -Deuda \\
\hline [V1] & IP - PPE & $0,2050^{* * *}$ & $-0,1228^{* * *}$ & 0,0934 & $-0,0524$ \\
[V2] & IP - PPE & 0,0713 & $0,2644^{* *}$ & $0,2808^{* * *}$ & 0,0966 \\
[V3] & IP - PPE & $-0,0739$ & $0,2038^{* *}$ & 0,1102 & 0,1186 \\
\hline
\end{tabular}

*** Diferencia estadísticamente significativo al $1 \%$; ** al $5 \%$; al $10 \%$

Panel B: Modelo [P2] a [P6] -requieren estimaciones de coeficientes de predicción-

\begin{tabular}{|c|c|c|c|c|c|c|c|}
\hline \multirow[t]{2}{*}{ Modelo } & \multirow[t]{2}{*}{ Muestra } & \multicolumn{2}{|c|}{$\begin{array}{l}\text { Mediana del error } \\
\text { absoluto de predicción } \\
\text { a } 1 \text { año } \\
\text { (sección cruzada global } \\
\text { para toda la muestra) }\end{array}$} & \multicolumn{2}{|c|}{$\begin{array}{l}\text { Mediana del error } \\
\text { absoluto de } \\
\text { predicción a } 1 \text { año } \\
\text { (sección cruzada por } \\
\text { submuestras) }\end{array}$} & \multicolumn{2}{|c|}{$\begin{array}{l}\text { Mediana del error } \\
\text { absoluto de } \\
\text { predicción a } 1 \text { año } \\
\text { (serie temporal) }\end{array}$} \\
\hline & & +Tamaño & -Tamaño & +Tamaño & -Tamaño & +Tamaño & -Tamaño \\
\hline [V4] & IP-PPE & 0,0243 & $-0,0090$ & 0,0266 & 0,0125 & $-0,1234$ & 0,0489 \\
\hline \multirow[t]{2}{*}{ [V5] } & IP-PPE & 0,0264 & $-0,0220$ & 0,0348 & 0,0045 & 0,0313 & $-0,1147$ \\
\hline & & + Deuda & -Deuda & + Deuda & -Deuda & + Deuda & -Deuda \\
\hline [V4] & IP-PPE & $0,1920 * *$ & $-0,0988$ & $0,1291^{*}$ & $-0,0663$ & 0,1138 & $-0,0883$ \\
\hline [V5] & IP-PPE & 0,0769 & $-0,0738$ & 0,0960 & $-0,0172$ & 0,1168 & $-0,2413^{*}$ \\
\hline
\end{tabular}

*** Diferencia estadísticamente significativo al $1 \% ; * *$ al $5 \% ; *$ al $10 \%$ 